\title{
Emission of Positron Annihilation Line Radiation by Clusters of Galaxies
}

\section{Citation}

Furlanetto, Steven R., and Abraham Loeb. 2002. "Emission of Positron Annihilation Line Radiation by Clusters of Galaxies." The Astrophysical Journal 572 (2): 796-809. https:// doi.org/10.1086/340384.

\section{Permanent link}

http://nrs.harvard.edu/urn-3:HUL.InstRepos:41393316

\section{Terms of Use}

This article was downloaded from Harvard University's DASH repository, and is made available under the terms and conditions applicable to Other Posted Material, as set forth at http:// nrs.harvard.edu/urn-3:HUL.InstRepos:dash.current.terms-of-use\#LAA

\section{Share Your Story}

The Harvard community has made this article openly available.

Please share how this access benefits you. Submit a story.

Accessibility 


\title{
EMISSION OF POSITRON ANNIHILATION LINE RADIATION BY CLUSTERS OF GALAXIES
}

\author{
Steven R. Furlanetto and Abraham Loeb \\ Harvard-Smithsonian Center for Astrophysics, 60 Garden Street, Cambridge, MA 02138; sfurlanetto@cfa.harvard.edu, aloeb@cfa.harvard.edu \\ Received 2002 January 18; accepted 2002 February 22
}

\begin{abstract}
Clusters of galaxies are enriched with positrons from jets of active galactic nuclei (AGNs) or from the interaction of cosmic rays with the intracluster gas. We follow the cooling of these positrons and show that their eventual annihilation with cluster electrons yields a narrow annihilation line. Unlike annihilation in the interstellar medium of galaxies, the line produced in clusters is not smeared by three-photon decay of positronium because positronium formation is suppressed at the high $(\gtrsim 1 \mathrm{keV})$ temperature of the cluster electrons. We show that if AGN jets are composed of $e^{+} e^{-}$pairs, then the annihilation line from rich clusters within a distance of $100 \mathrm{Mpc}$ might be detectable with future space missions, such as the International Gamma-Ray Astrophysical Laboratory or the Energetic X-Ray Imaging Survey Telescope.
\end{abstract}

Subject headings: cosmic rays — elementary particles — galaxies: clusters: general — galaxies: jets — $\mathrm{X}$-rays: galaxies: clusters

\section{INTRODUCTION}

Despite decades of intense study, the composition of relativistic radio jets remains enigmatic. While the existence of synchrotron-emitting electrons is secure in both quasars (Begelman, Blandford, \& Rees 1984) and microquasars (Mirabel 2001), there is no conclusive evidence that can determine whether positrons or protons make up the positively charged component. Naively, one might expect these cases to be easily distinguishable through an observational search for the electron-positron annihilation line. However, modeling of the annihilation process in active jets has shown this not to be the case: the annihilation spectral feature is not a line but is instead very broad and hence difficult to identify unambiguously (although it may possibly contribute to the observed $\gamma$-ray spectrum of blazars; see Boettcher \& Schlickeiser 1996). Even if positron cooling to nonrelativistic energies within the jet is efficient, the high bulk Lorentz factor $\left(\gamma_{\text {jet }} \sim 10\right)$ of the jet would likely smear out an annihilation line (Begelman et al. 1984). The resulting radiation may be detectable with upcoming space observatories, although its interpretation will depend critically on proper modeling of the region inside the jet (Wang, Durouchoux, \& Li 2001).

The chief obstacle to producing a recognizable annihilation line feature is the highly relativistic nature of the associated plasma. This obstacle may be overcome simply by waiting for the active galactic nucleus (AGN) to become dormant. Once the central engine disappears, the material in the jet will presumably mix with the surrounding medium and cool to the ambient temperature. In this paper, we calculate the annihilation signal from "relic" positrons produced by an AGN embedded in a galaxy cluster as the positrons thermalize with the electrons in the intracluster medium (ICM). We show that under reasonable assumptions, an annihilation line from nearby clusters will be observable in the near future.

A similar suggestion of thermal annihilation after escape from the accelerating source was made by Maciolek-Niedzwiecki, Zdziarski, \& Coppi (1995) in the context of smallscale jets from stellar-mass black holes. However, in galactic environments with typical temperatures $T \lesssim 10^{6} \mathrm{~K}$, formation of an annihilation line at $511 \mathrm{keV}$ is inhibited by the rapid formation of positronium, whose primary annihilation channel yields three photons. For example, the observations of Kinzer et al. (2001) show that $93 \% \pm 4 \%$ of pair annihilations in the Galactic center occur through the positronium channel and that $\gtrsim 70 \%$ of the total annihilation energy is emitted in a broad continuum rather than in the line. We will argue that galaxy clusters are ideally suited to producing annihilation lines because the characteristic temperature of the ICM is larger than the binding energy of positronium; hence, nearly all annihilations produce photons near $511 \mathrm{keV}$.

Another important open question involves the dynamical significance of cosmic rays to the ICM. Faraday rotation studies have revealed that cluster cores have magnetic fields ¿1 $\mathrm{G}$ (Kim et al. 1990; Clarke, Kronberg, \& Böhringer 2001), while observations of excess emission in the ultraviolet and hard X-ray bands from the ICM have been used to infer the existence of widespread cluster fields at slightly lower levels $\sim 0.1-1 \mu \mathrm{G}$ (e.g., Rephaeli, Gruber, \& Blanco 1999; Fusco-Femiano et al. 1999). The pervasiveness of magnetic fields in clusters indicates that collisionless shocks generated by accretion or merger events may be efficient particle accelerators (Blandford \& Eichler 1987; Colafrancesco \& Blasi 1998). Observations of synchrotron radio halos indicate that the acceleration of electrons by cluster merger shocks is a common occurrence (Kempner \& Sarazin 2001). However, these observations probe only the electron component. In order to understand the dynamical effects of the cosmic rays on the cluster, we are most interested in the accelerated protons, both because shock acceleration may inject more energy into this component than into the electron component (Fields et al. 2001; Butt et al. 2001) and because cosmic-ray protons do not lose their energy rapidly through radiative cooling.

Recent cosmological simulations suggest that the cosmicray pressure might be as large as $\sim 10 \%-40 \%$ of the thermal gas pressure in clusters (Miniati et al. 2001b). To date, two diagnostics of the cosmic-ray proton content have been proposed, both relying on the decay of pions produced in colli- 
sions between cosmic-ray and thermal protons, namely, synchrotron and inverse Compton emission from secondary electrons and positrons produced in charged pion decay (Blasi \& Colafrancesco 1999; Dolag \& Enßlin 2000; Miniati et al. 2001a) and $\gamma$-rays produced in neutral pion decay (Colafrancesco \& Blasi 1998; Blasi 1999; Miniati et al. 2001b). The former process suffers from the possibility of contamination by newly accelerated electrons, while the $\mathrm{GeV} \gamma$-ray signal will remain below the detection limits at least until the launch of the Gamma Ray Large Area Space Telescope mission ${ }^{1}$ in 2005, largely because the energy from the decaying pions is distributed over a very wide range of photon energies. It may, however, be detectable by existing or upcoming $\mathrm{TeV} \gamma$-ray detectors (Blasi 1999).

Because of the rapid cooling of positrons produced in the decay of $\pi^{+}$to nonrelativistic temperatures, we would expect an annihilation line to be produced in this case as well. (Here, too, the formation of positronium is inhibited by the relatively large cluster temperatures.) We therefore also calculate the annihilation spectrum produced through secondary positron production by cosmic-ray protons. However, we find that this signal is well below the detection thresholds of upcoming instruments. Hence, AGNs are the only realistic pollutant of substantial amounts of positrons into galaxy clusters. We thus argue that the future detection of positron annihilation line radiation from clusters would constitute a robust signature of electron-positron jets.

We begin by describing the factors determining the evolution of the positron population in $\S 2$, including cooling, annihilation, and source terms. We then solve the evolution equations in $\S 3$. In $\S 4$, we calculate the resulting positron annihilation signals. Finally, we conclude in $\S 5$ with a discussion of our results and prospects for future observations.

Throughout the paper, we assume a $\Lambda \mathrm{CDM}$ cosmology with $\Omega_{0}=0.3, \Omega_{\Lambda}=0.7$, and $H_{0}=70 \mathrm{~km} \mathrm{~s}^{-1} \mathrm{Mpc}^{-1}$.

\section{EVOLUTION OF THE POSITRON DISTRIBUTION FUNCTION}

The time evolution of $N_{+}(\gamma, t)$, the mean differential number density of positrons with Lorentz factor between $(\gamma, \gamma+d \gamma)$ in the cluster at time $t$, is described by the ana$\log$ of the Boltzmann equation with source and sink terms (Sarazin 1999):

$$
\begin{aligned}
\frac{\partial N_{+}(\gamma, t)}{\partial t}= & \frac{\partial}{\partial \gamma}\left[b(\gamma, t) N_{+}(\gamma, t)\right] \\
& +Q(\gamma)-N_{+}(\gamma, t) A(\gamma) .
\end{aligned}
$$

Here, $b(\gamma, t)=(d \gamma / d t)$ is the cooling rate of a single positron, $Q(\gamma) d \gamma$ is the production rate of positrons per unit volume in the interval $(\gamma, \gamma+d \gamma)$, and $A(\gamma) d \gamma$ is the annihilation rate of positrons in the same interval. We have neglected diffusion and loss of positrons between volume elements in the ICM (Ginzburg \& Syrovatskii 1964). This should be a good approximation, provided that the sources of positrons are distributed over a sufficiently large volume, as would be expected for injection by both AGNs and cluster shocks. Alternatively, because clusters trap all but the very highest energy cosmic rays over a Hubble time (Bere-

\footnotetext{
${ }^{1}$ See http://www-glast.stanford.edu/mission.html.
}

zinsky, Blasi, \& Ptuskin 1997), our evolution equation can be viewed as simply describing the total positron population in the cluster, with the appropriate averages of the cooling, source, and annihilation terms (Sarazin 1999).

We are concerned primarily with those positrons that cool sufficiently to contribute to line emission upon annihilation. Therefore, we wish to track the number of positrons that have thermalized with the ambient electrons. In the test particle approximation, a positron cools until its kinetic energy $\epsilon_{K} \approx 0.98 k_{\mathrm{B}} T_{e}$, where $k_{\mathrm{B}}$ is Boltzmann's constant and $T_{e}$ is the temperature of the ambient thermal electrons (Trubnikov 1965). In actuality, the finite population of cooled positrons thermalizes with the ambient electrons on a timescale (Trubnikov 1965)

$$
\tau_{\text {therm }} \approx 4.8 \times 10^{3} T_{\mathrm{keV}}^{3 / 2} \frac{10^{-3} \mathrm{~cm}^{-3}}{n_{e}} \mathrm{yr},
$$

where $n_{e}$ is the electron number density in the cluster core and $T_{\mathrm{keV}}=\left(k_{\mathrm{B}} T_{e} / \mathrm{keV}\right)$. Because $\tau_{\text {therm }}$ is much shorter than the other relevant timescales, we assume that thermalization occurs instantaneously. The pool of cooled positrons essentially acts as an absorbing wall in the cooling equation, so we write the total positron distribution function $N_{\mathrm{T}}(\gamma)$ as

$$
N_{\mathrm{T}}(\gamma, t)=n_{+}(t) \delta\left(\gamma-\gamma_{\mathrm{eq}}\right)+N_{+}(\gamma, t)
$$

where $\gamma_{\mathrm{eq}}$ is the mean Lorentz factor of the ambient electrons and $n_{+}$is the number density of the thermalized positrons.

In the following subsections, we describe each of the terms on the right-hand side of equation (1) in detail. We solve for the $N_{+}(\gamma, t)$ appropriate to our two models in $\S 3$.

\subsection{Loss Rate $b(\gamma, t)$}

The principal cooling mechanisms for cosmic-ray electrons or positrons in a cluster are synchrotron, inverse Compton (IC), and Coulomb cooling (Rephaeli 1979). The synchrotron and IC loss rates for a single positron with Lorentz factor $\gamma \gg 1$ are given by (Rephaeli 1979)

$$
\left(\frac{d \gamma}{d t}\right)_{\mathrm{syn}, \mathrm{IC}}=-\frac{4 \sigma_{\mathrm{T}}}{3 m_{e} c}\left(u_{\mathrm{B}}+u_{\mathrm{CMB}}\right) \gamma^{2},
$$

where $\sigma_{\mathrm{T}}$ is the Thomson cross section, $m_{e}$ is the electron mass, $u_{\mathrm{B}}=B^{2} / 8 \pi$ is the energy density in the cluster magnetic field, and $u_{\mathrm{CMB}}$ is the energy density of the cosmic microwave background (CMB). The loss rate due to these two processes is therefore

$$
\begin{array}{r}
b_{\text {syn }, \mathrm{IC}}(\gamma, t)=-\left(\frac{d \gamma}{d t}\right)_{\text {syn }, \mathrm{IC}}=4.3 \times 10^{-13} \gamma^{2} \\
\times\left[(1+z)^{4}+0.86\left(\frac{B}{3 \mu \mathrm{G}}\right)^{2}\right] \mathrm{yr}^{-1},
\end{array}
$$

where $z$ is the cosmological redshift at cosmic time $t$.

The loss rate from Coulomb cooling is approximately (Sarazin \& Kempner 2000)

$$
\left(\frac{d \gamma}{d t}\right)_{\mathrm{Coul}}=-\frac{4 \pi n_{e} e^{4}}{\beta m_{e}^{2} c^{3}} \ln \left(\frac{1.12 m_{e} c^{2} \gamma^{1 / 2} \beta^{2}}{\hbar \omega_{p}}\right),
$$


where $\beta=\left(1-\gamma^{-2}\right)^{1 / 2}$ is the positron speed normalized to the speed of light and $\omega_{p}$ is the plasma frequency of the ambient electron gas. The corresponding choice for the Coulomb logarithm is valid for $\alpha \ll \beta \ll 1$, where $\alpha$ is the fine structure constant, and for $\gamma \gg 1$, with errors $\lesssim 10 \%$ in the intermediate regime (Sarazin \& Kempner 2000). Evaluating the constants, we find

$$
\begin{aligned}
b_{\text {Coul }}(\gamma)= & -\left(\frac{d \gamma}{d t}\right)_{\text {Coul }}=3.5 \times 10^{-5} \frac{n_{\mathrm{cm}}}{\beta} \\
& \times\left[1+\frac{1}{74.5} \ln \left(\frac{\gamma \beta^{4}}{n_{\mathrm{cm}}}\right)\right] \mathrm{yr}^{-1},
\end{aligned}
$$

where $n_{\mathrm{cm}}=\left(n_{e} / \mathrm{cm}^{-3}\right)$.

The total loss rate is then $b(\gamma, t)=b_{\text {Coul }}(\gamma)+$ $b_{\text {syn,IC }}(\gamma, t)$. Comparing equations (5) and (7), we see that IC/synchrotron cooling dominates for $\gamma \gtrsim 200\left(n_{e} / 10^{-3}\right.$ $\left.\mathrm{cm}^{-3}\right)^{1 / 2}$; hence, our assumption that $\gamma \gg 1$ in equation (4) is appropriate.

\subsection{Annihilation Rate $A(\gamma)$}

The annihilation rate $A\left(\gamma_{+}\right)$of positrons at a given Lorentz factor through the process $e^{+} e^{-} \rightarrow 2 \gamma$ may be written as (Svensson 1982)

$$
A\left(\gamma_{+}\right)=\int_{1}^{\infty} d \gamma_{-} N_{-}\left(\gamma_{-}\right) \int_{-1}^{1} \frac{d \mu}{2} \frac{\gamma_{\mathrm{CM}}^{2}}{\gamma_{+} \gamma_{-}} 2 \beta_{\mathrm{CM}} c \sigma_{e e}\left(\gamma_{\mathrm{CM}}\right),
$$

assuming that the distribution function of electrons, $N_{-}\left(\gamma_{-}\right)$, is isotropic but otherwise arbitrary. Here, $\gamma_{+}$ $\left(\gamma_{-}\right)$is the Lorentz factor of the positron (electron) in the cluster rest frame, $\gamma_{\mathrm{CM}}$ is the Lorentz factor of the positron in the center-of-mass (CM) frame, $\mu$ is the interaction angle in the cluster rest-frame, and $\sigma_{e e}\left(\gamma_{\mathrm{CM}}\right)$ is the total annihilation cross section in the CM frame. We find that assuming that the ambient electrons are all at rest $\left[N_{-}\left(\gamma_{-}\right)=n_{e} \delta\left(\gamma_{-}-1\right)\right]$ makes only a small difference to the total annihilation cross section for the nonthermalized positrons because realistic cluster temperatures are highly nonrelativistic (so that $\gamma_{+} \gg \gamma_{-}$). In this cosmic-ray approximation, $\gamma_{\mathrm{CM}}^{2}=\left(\gamma_{+}+1\right) / 2$ and the annihilation rate becomes

$$
\begin{aligned}
A\left(\gamma_{+}\right)=\frac{\pi c n_{e} r_{e}^{2} \beta_{+}}{\gamma_{+}+1}\left\{\frac{\gamma_{+}^{2}+4 \gamma_{+}+1}{\gamma_{+}^{2}-1}\right. & \ln \left[\gamma_{+}+\left(\gamma_{+}^{2}-1\right)^{1 / 2}\right] \\
& \left.-\frac{\gamma_{+}+3}{\left(\gamma_{+}^{2}-1\right)^{1 / 2}}\right\},
\end{aligned}
$$

where $r_{e}$ is the classical electron radius. This expression is valid provided Coulomb corrections may be neglected, i.e., as long as $\beta_{+} \gg \alpha$; this condition is satisfied for all $\gamma_{+} \geq \gamma_{\text {eq }}$ at the typical cluster temperatures of $k_{\mathrm{B}} T_{e} \gtrsim 1 \mathrm{keV}$.

In order to calculate the spectrum of annihilation photons, we are also interested in the derivative of the annihilation rate with respect to photon energy. This may be written in the cosmic-ray approximation as (Svensson 1982)

$$
\frac{d n_{\gamma}}{d k d t}=n_{e} \int_{1}^{\infty} d \gamma_{+} N\left(\gamma_{+}\right)\left\langle v \frac{d \sigma}{d k}\left(k, \gamma_{+}\right)\right\rangle
$$

where $k \equiv \epsilon_{\gamma} / m_{e} c^{2}$ is the normalized photon energy and

$$
\begin{aligned}
& \left\langle v \frac{d \sigma}{d k}\left(k, \gamma_{+}\right)\right\rangle \\
& =\frac{\pi c r_{e}^{2}}{\beta_{+} \gamma_{+}^{2}}\left\{\frac{-\left(3+\gamma_{+}\right) /\left(1+\gamma_{+}\right)+\left(3+\gamma_{+}\right) / k-1 / k^{2}}{\left[1-k /\left(1+\gamma_{+}\right)\right]^{2}}-2\right\},
\end{aligned}
$$

is the angle-averaged emissivity per electron-positron pair.

For the thermalized positrons, the cosmic-ray approximation is invalid because $\gamma_{+} \approx \gamma_{-}$. We must therefore evaluate the general expression for the photon production rate

$$
\frac{d n_{\gamma}}{d k d t}=\int_{1}^{\infty} d \gamma_{+} N\left(\gamma_{+}\right) \int_{1}^{\infty} d \gamma_{-} N_{-}\left(\gamma_{-}\right)\left\langle v \frac{d \sigma}{d k}\left(k, \gamma_{+}, \gamma_{-}\right)\right\rangle,
$$

for Maxwell-Boltzmann electron and positron distributions at a temperature $T_{e}$. Svensson, Larsson, \& Poutanen (1996) show that the result is

$$
\begin{aligned}
\left.\frac{d n_{\gamma}}{d k d t}\right|_{\text {line }}= & \frac{3}{4 \sqrt{\pi}} n_{+} n_{e} c \sigma_{\mathrm{T}} k^{3 / 2}\left(\frac{m_{e} c^{2}}{k_{\mathrm{B}} T_{e}}\right)^{1 / 2} \\
& \times \exp \left[-\frac{(k-1)^{2}}{\left(k_{\mathrm{B}} T_{e} / m_{e} c^{2}\right) k}\right],
\end{aligned}
$$

in the approximation $k \ll 1$ and $k_{\mathrm{B}} T_{e} \ll m_{e} c^{2}$. Here, we use the subscript "line" because annihilation of the thermalized component yields a well-defined spectral line (see $\S 4$ below). The total annihilation rate of the thermalized positrons is then simply

$$
\begin{aligned}
A\left(\gamma_{\mathrm{eq}}\right) & =\left.\frac{1}{2 n_{+}} \int d k \frac{d n_{\gamma}}{d k d t}\right|_{\text {line }} \\
& \approx 8 \times 10^{-15} n_{e} \mathrm{~s}^{-1},
\end{aligned}
$$

with only a very weak dependence on temperature in the range of interest. (The factor of 2 in the denominator appears because each annihilating positron yields two photons.)

Note that in many astrophysical situations, positron annihilation occurs principally through the formation and subsequent decay of positronium. In such situations, the dominant annihilation mechanism is the decay of orthopositronium, which must emit at least three photons in order to conserve angular momentum (Berestetskii, Lifshitz, \& Pitaevskii 1982). This mechanism therefore precludes the formation of an annihilation line. Fortunately, in our case positronium formation is insignificant. For temperatures $k_{\mathrm{B}} T_{e} \gtrsim 1 \mathrm{keV}$, the cross section for positronium formation amounts to $\lesssim 10 \%$ of the cross section for direct annihilation, and the positronium formation rate decreases strongly at yet higher temperatures (Crannell et al. 1976). We therefore neglect this annihilation channel in our calculations. Note, however, that in cool galactic environments $\left(T \lesssim 10^{5} \mathrm{~K}\right)$, annihilation through positronium formation dominates and the annihilation line is suppressed.

\subsection{Source Function $Q(\gamma)$}

In the case of positron injection purely via an AGN outflow, the source function $Q(\gamma)$ is nonzero only at the instant 
of injection. In this section, we describe positron production mechanisms in galaxy clusters over and above direct injection from AGNs. The dominant mechanism, the protonproton channel, is described in $\S 2.3 .1$. For completeness, we examine other mechanisms in $\S 2.3 .2$.

\subsubsection{Positron Production from Proton-Proton Collisions}

Collisions between thermal and cosmic-ray protons produce positrons primarily through the reaction chain

$$
\begin{aligned}
p+p & \rightarrow \pi^{+}+X, \\
\pi^{+} & \rightarrow \mu^{+}+\nu_{\mu}, \\
\mu^{+} & \rightarrow e^{+}+\bar{\nu}_{\mu}+\nu_{e},
\end{aligned}
$$

provided that the cosmic-ray proton has a Lorentz factor exceeding $\gamma_{\text {th }}=1.3$, the threshold for pion production (Mannheim \& Schlickeiser 1994). The resulting positron production rate is then (Moskalenko \& Strong 1998)

$$
\begin{aligned}
Q_{\mathrm{pp}}(\gamma)= & n_{\mathrm{H}} \int_{\gamma_{p}^{\min }}^{\infty} d \gamma_{p} J_{p}\left(\gamma_{p}\right) \sigma_{\pi^{+}}\left(\gamma_{p}\right) \\
& \times \int_{\gamma_{\pi}^{\min }}^{\gamma_{\pi}^{\max }} d \gamma_{\pi} F_{+}\left(\gamma, \gamma_{\pi}\right) F_{\pi}\left(\gamma_{\pi}, \gamma_{p}\right),
\end{aligned}
$$

where $n_{\mathrm{H}}=n_{e}$ is the proton density in the cluster core, $J_{p}\left(\gamma_{p}\right)$ is the differential cosmic-ray proton flux at Lorentz factor $\gamma_{p}, \sigma_{\pi^{+}}\left(\gamma_{p}\right)$ is the cross section for $\pi^{+}$production in the collision (including all channels), $F_{+}\left(\gamma, \gamma_{\pi}\right)$ is the normalized positron distribution function at positron Lorentz factor $\gamma$ for positrons produced by the decay of a pion with Lorentz factor $\gamma_{\pi}$, and $F_{\pi}\left(\gamma_{\pi}, \gamma_{p}\right)$ is the normalized pion distribution function produced by a collision between a thermal proton and a cosmic-ray proton with Lorentz factor $\gamma_{p}$. The maximum cosmic-ray energy $\gamma_{p}^{\max }$ is determined below. The other integration limits are determined by kinematics: $\gamma_{\pi}^{\min }$ is the minimum pion Lorentz factor needed to produce a positron with $\gamma, \gamma_{\pi}^{\max }$ is the maximum pion Lorentz factor that can be produced by a proton with $\gamma_{p}$, and $\gamma_{p}^{\min }$ is the minimum proton Lorentz factor required to produce a positron with $\gamma$.

Our calculation of the resulting distribution function follows closely the method outlined in Appendices B and C of Moskalenko \& Strong (1998), so we only summarize it here. Unfortunately, there is no firm theoretical understanding of pion production in proton-proton collisions. The production cross sections are experimentally well determined (see the compilation of Dermer 1986), but the resulting $F_{\pi}\left(\gamma_{\pi}, \gamma_{p}\right)$ are not. Instead, we must use approximate models of the interaction. At low energies $\left(\gamma_{p} \lesssim 3\right)$, the "isobaric model" of Stecker (1970), in which the collision forms a $\Delta$ isobar that subsequently decays into a pion, fits the data best, while at high energies $\left(\gamma_{p} \gtrsim 7\right)$, the "scaling model" of Badhwar, Golden, \& Stephens (1977) provides a better fit. In the intermediate regime, we take a linear interpolation between the two models. These models do not include the deuterium channel $\left(p p \rightarrow D \pi^{+}\right)$, important for low-energy collisions, and we include this separately (note that this channel can be treated exactly). We assume that $\mu^{+}$are produced isotropically in the $\pi^{+}$decay, and we include polarization in the subsequent $\mu^{+}$decay (see Moskalenko \& Strong 1998 for details). Note that $F_{+}\left(\gamma, \gamma_{\pi}\right)$ can be found exactly, unlike the pion distribution function.

Our treatment neglects two effects in pion production. First, we ignore the kaon channel, in which the collision forms a $K^{+}$that subsequently decays into either a $\pi^{+}$or directly into a $\mu^{+}$. Miniati (2001) shows that the kaon channel provides only a small correction to the dominant pion channel except at very high secondary energies (where the total number of particles is very small regardless). Second, we assume that the ICM is composed entirely of hydrogen, while in reality $\sim 25 \%$ of the mass is contained in helium nuclei. Mannheim \& Schlickeiser (1994) show that modifying the ambient medium to a normal interstellar medium composition increases the $\pi$ production rate by $\lesssim 30 \%$.

The production rate depends critically on the input flux of cosmic-ray protons:

$$
J_{p}\left(\gamma_{p}\right)=\frac{c}{4 \pi} \beta\left(\gamma_{p}\right) N_{p}\left(\gamma_{p}\right)
$$

where $N_{p}\left(\gamma_{p}\right)$ is the differential number density of cosmicray protons with Lorentz factors between $\left(\gamma_{p}, \gamma_{p}+d \gamma_{p}\right)$. The protons are expected to be accelerated either by the accretion shock surrounding the cluster or by shocks generated during merger events. Such shocks are collisionless and nonrelativistic and hence could efficiently accelerate particles to relativistic energies (Bell 1978; Blandford \& Ostriker 1978; Blandford \& Eichler 1987), as observed locally in supernova remnants (Koyama et al. 1995, 1997; Tanimori et al. 1998; Muraishi et al. 2000; Butt et al. 2001).

We normalize the proton number density by requiring that the kinetic energy density in relativistic protons be a fraction $\xi_{\mathrm{cr}}$ of the total thermal energy density $u_{\mathrm{th}}=3 n_{e} k_{\mathrm{B}} T$ of the cluster:

$$
m_{p} c^{2} \int\left(\gamma_{p}-1\right) N_{p}\left(\gamma_{p}\right) d \gamma_{p}=\xi_{\mathrm{cr}} u_{\mathrm{th}} .
$$

Shock-acceleration models predict that the distribution function of relativistic particles is $\propto p^{-s}$, where $p$ is the particle momentum, $s=(r+2) /(r-1)$, and $r$ is the shock compression ratio (Blandford \& Eichler 1987). The differential number density of accelerated protons may therefore be written as

$N_{p}\left(\gamma_{p}\right)=N_{p, 0} \frac{\gamma_{p}}{\left(\gamma_{p}^{2}-1\right)^{(s+1) / 2}} \quad \gamma_{p, \min }<\gamma_{p}<\gamma_{p, \max }$

where $\gamma_{p \text {,min }}$ and $\gamma_{p \text {,max }}$ are the minimum and maximum Lorentz factor to which a proton can be accelerated in the cluster shocks. For strong shocks in a gas with adiabatic index $\Gamma=5 / 3, s=2$. Miniati et al. (2001b) argue that the accelerating shocks in cluster environments are relatively weak, with Mach numbers $\lesssim 4-5$, corresponding to power-law indices $s \gtrsim 2.25$. We consider the two cases $s=2$ and $s=3$ in the following; these should bracket the (uncertain) distribution in cluster environments. The normalization constant $N_{p, 0}$ is

$$
N_{p, 0}=\frac{3.2 \times 10^{-7}}{C_{S}^{\mathrm{cr}}} n_{e} T_{\mathrm{keV}} \frac{\xi_{\mathrm{cr}}}{0.1} \mathrm{~cm}^{-3},
$$

where $C_{S}^{\mathrm{cr}}$ depends on the power-law index of the cosmic- 
ray distribution function through equation (17); for example,

$$
\begin{aligned}
C_{2}^{\mathrm{cr}}= & \ln \left(\frac{\gamma_{p, \text { max }}+\sqrt{\gamma_{p, \text { max }}^{2}-1}}{\gamma_{p, \min }+\sqrt{\gamma_{p, \min }^{2}-1}}\right) \\
& +\frac{\sqrt{\gamma_{p, \min }^{2}-1}}{\gamma_{p, \min }+1}-\frac{\sqrt{\gamma_{p, \max }^{2}-1}}{\gamma_{p, \max }+1}
\end{aligned}
$$

while

$$
\begin{aligned}
C_{3}^{\mathrm{cr}}= & \frac{1}{4}\left\{\ln \left[\frac{\left(\gamma_{p, \max }-1\right)\left(\gamma_{p, \min }+1\right)}{\left(\gamma_{p, \max }+1\right)\left(\gamma_{p, \min }-1\right)}\right]\right. \\
& \left.+\frac{2}{\gamma_{p, \min }+1}-\frac{2}{\gamma_{p, \max }+1}\right\} .
\end{aligned}
$$

Our fiducial value of $\xi_{\text {cr }}=0.1$ is probably a lower limit to the energy deposited in cosmic-ray protons (Fields et al. 2001; Butt et al. 2001).

The maximum energy to which protons may be accelerated is relatively well determined. Two factors limit $\gamma_{p \text {,max }}$ : the acceleration time $t_{\mathrm{acc}} \sim r_{\mathrm{L}} c / v_{s}^{2}$ must be smaller than both the age of the cluster and the cooling timescale; here $v_{s}$ is the shock velocity (assumed to be close to the sound speed in the cluster) and $r_{\mathrm{L}}$ is the proton Larmor radius. The cooling time of the protons (which is determined primarily by pion production) exceeds the age of the universe, so the first condition is the critical one, yielding $\gamma_{p \text {, max }} \sim 5 \times$ $10^{9} B_{\mu \mathrm{G}} T_{\mathrm{keV}}$, where $B_{\mu \mathrm{G}}$ is the shock magnetic field in microgauss. Note that because $\gamma_{p, \max } \gg 1, C_{3}^{\mathrm{cr}}$ is essentially independent of its precise value, while it enters $C_{2}^{\text {cr }}$ only logarithmically.

The value of $\gamma_{p \text {,min }}$ is less certain. Most analytic models of diffusive shock acceleration assume that the particles retain a Maxwell-Boltzmann distribution below the minimum injection momentum $p_{\min }$ and a (broken) power law above that value (e.g., Kang \& Jones 1995; Ellison, Berezhko, \& Baring 2000). Normally, $p_{\min }$ is a few times the mean thermal momentum behind the accelerating shock, i.e., $p_{\text {min }} \approx 2 c_{1}\left(m_{p} k_{\mathrm{B}} T_{\mathrm{sh}}\right)^{1 / 2}$, where $T_{\mathrm{sh}}$ is the postshock temperature. In principle, $c_{1} \sim 2-3$ is determined by the assumed energy density of the cosmic-ray component. However, because $p_{\min }$ is located in the exponential tail of the Maxwellian distribution, it depends only very weakly on the cosmicray energy density. We therefore fix $c_{1} \sim 2.3$, a value inferred from numerical simulations of the shock-acceleration process (Gieseler, Jones, \& Kang 2000). Then

$$
\gamma_{p, \min }-1 \approx 3.4 \times 10^{-5} T_{\mathrm{keV}}\left(\frac{c_{1}}{2.3}\right)^{2}\left(\frac{M}{4}\right)^{2}
$$

where $M$ is the mean Mach number of the shocks. In our calculations, we fix $M=4$, as suggested by Miniati et al. (2001b). Note that particularly in the $s=3$ case, a nonnegligible fraction of the cosmic-ray energy may be carried by protons with $\gamma_{p}<\gamma_{\text {th }}$.

The positron production rate via proton collisions $Q_{\mathrm{pp}}(\gamma)$ is shown in Figure 1 for input proton spectra with $s=2$ (solid curves) and with $s=3$ (dotted curves). Because $Q_{\mathrm{pp}}$ is

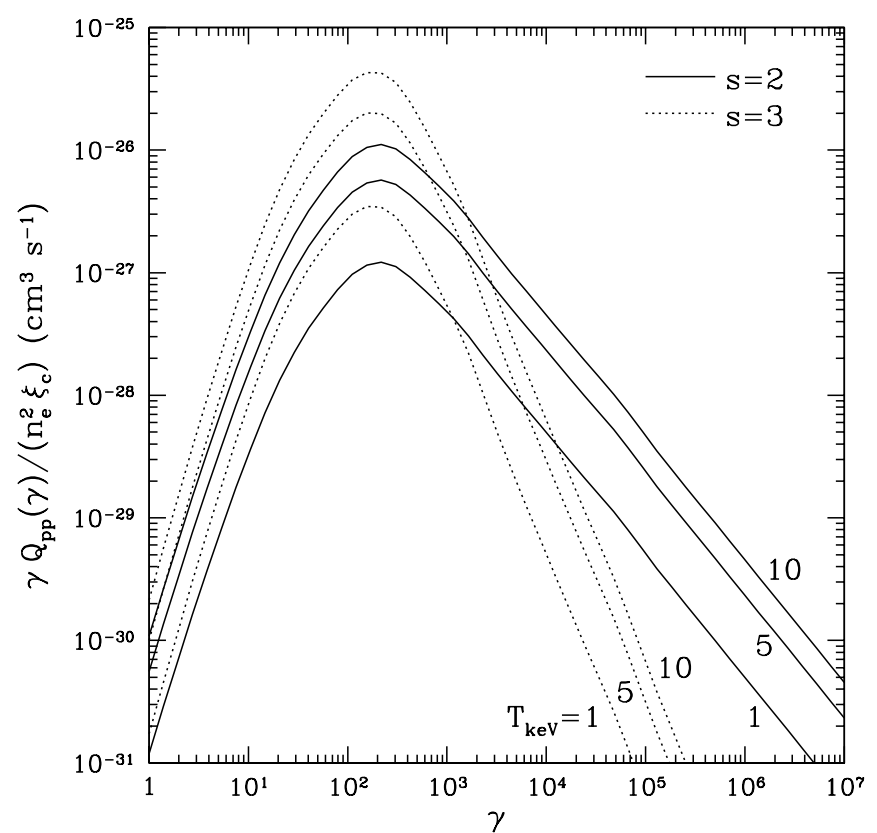

FIG. 1.-Normalized positron production spectrum from proton-proton collisions, $Q_{\mathrm{pp}}(\gamma) /\left(n_{e}^{2} \xi_{\mathrm{cr}}\right)$. Shown are source functions for an $s=2$ (solid curves) and an $s=3$ (dotted curves) cosmic-ray proton spectrum. In each case, the curves assume $k_{\mathrm{B}} T_{e}=1,5$, and $10 \mathrm{keV}$, from bottom to top.

strictly proportional to $\xi_{\mathrm{cr}} n_{e}^{2}$, we normalize our results by this quantity. The dependence on $T_{e}$ is slightly more subtle, so we show results for $k_{\mathrm{B}} T_{e}=1,5$, and $10 \mathrm{keV}$, from bottom to top. The ambient temperature does not affect the shape of the positron spectrum (because in all cases $\left.\gamma_{p \text {, min }}<\gamma_{\text {th }}\right)$, but it does affect the overall normalization factor $N_{p, 0}$ (eq. [19]) and the fraction of cosmic rays with $\gamma_{p}>\gamma_{\text {th }}$ (eq. [22]). Nevertheless, our results differ only slightly from the $Q_{\mathrm{pp}} \propto T_{e}$ scaling that one would naively expect from equation (19). Figure 1 shows that the positrons are produced with a characteristic $\gamma_{+} \sim 200$; this indicates that $\gtrsim 99 \%$ of the energy deposited in positrons will be lost to cooling before annihilation. At high positron energies, $Q_{\mathrm{pp}} \propto \gamma^{-s}$. This occurs because $\sigma_{\pi^{+}}$is only a weak function of proton energy for $\gamma_{p} \gg 1$.

For reference, the total positron production rate via proton collisions is $Q_{\mathrm{pp}} \approx 4.5 \times 10^{-17} n_{e} N_{p, 0} \mathrm{~cm}^{-3} \mathrm{~s}^{-1}$ for an $s=2$ cosmic-ray spectrum and $Q_{\mathrm{pp}} \approx 1.5 \times 10^{-17} n_{e} N_{p, 0}$ $\mathrm{cm}^{-3} \mathrm{~s}^{-1}$ for an $s=3$ spectrum.

\subsubsection{Other Positron Production Mechanisms}

Cosmic-ray protons can create positrons through two additional channels, photopair production $(p+\gamma \rightarrow p+$ $\left.e^{+}+e^{-}\right)$and photopion production $\left(p+\gamma \rightarrow n+\pi^{+}\right)$followed by $\pi^{+}$decay. In the diffuse IGM, these processes are unimportant except for the highest energy cosmic rays because photopair (or photopion) production requires $\gamma_{p} \gtrsim 7 \times 10^{8}$ (or $\gamma_{p} \gtrsim 10^{11}$ ) if the photon originates from the $\mathrm{CMB}$. However, the high-temperature radiation field in clusters lowers these thresholds dramatically to $\gamma_{p} \gtrsim 511 T_{\mathrm{keV}}^{-1}$ for photopair production and $\gamma_{p} \gtrsim 8 \times 10^{4} T_{\mathrm{keV}}^{-1}$ for photopion production. These channels could therefore in principle play an important role in positron production in galaxy clusters. The effects of the lowered thresholds have 
not to our knowledge been addressed explicitly in the literature, so we will estimate the total production rates here. We assume that the ambient photon field arises from bremsstrahlung radiation, with a differential energy density $u_{\nu}=4 \pi r_{c} \varepsilon_{\nu}^{f f} / 3 c$, where $r_{c}$ is the core radius of the cluster and $\varepsilon_{\nu}^{f f}$ is the bremsstrahlung spectral emissivity function (Rybicki \& Lightman 1979).

For either of these processes, the particle creation rate for a proton traveling with Lorentz factor $\gamma_{p}$ may be written as (Mannheim \& Schlickeiser 1994)

$$
\frac{d n_{+}}{d t}=\frac{c}{2 \gamma_{p}^{2}} \int_{x_{\mathrm{th}}^{\prime} / 2 \gamma_{p}}^{\infty} \frac{d x}{x^{2}} \frac{d n}{d x} \int_{x_{\mathrm{th}}^{\prime}}^{2 \gamma_{p} x} d x^{\prime} x^{\prime} \sigma\left(x^{\prime}\right),
$$

where $d n / d x$ is the (isotropic) photon distribution function, $\sigma$ is the relevant collision cross section, and $x_{\mathrm{th}}^{\prime}$ is the minimum photon energy in the proton rest frame needed to create the specified particle(s). If we approximate $\sigma$ as a constant and the exponential cutoff in the bremsstrahlung photon spectrum as a step function with cutoff $k_{\mathrm{B}} T_{e}$, then the integrals may be evaluated easily. We find that the positron creation rate due to a single proton is

$$
\frac{d n_{+}}{d t}=K c \sigma\left[\ln \left(\frac{\gamma_{p}}{\gamma_{*}}\right)+\frac{\gamma_{*}^{2}}{\gamma_{p}^{2}}-1\right],
$$

where $K$ is a constant evaluated below and $\gamma_{*}=x_{\mathrm{th}}^{\prime} / 2 k_{\mathrm{B}} T_{e}$ is the minimum proton Lorentz factor required to produce the particle(s) given our assumed photon spectrum.

If we assume a cosmic-ray spectrum $N_{p}\left(\gamma_{p}\right)=N_{p, 0} \gamma_{p}^{-2}$, maximizing the effects of protons with $\gamma_{p}>\gamma_{*}$, the total rate of positron production $Q$ is

$$
Q=\frac{K c \sigma N_{p, 0}}{3 \gamma_{*}} .
$$

For the photoproduction processes we consider, $\sigma_{\gamma e^{+} e^{-}} \sim 6$ mbarns (Chodorowski, Zdziarski, \& Sikora 1992), while $\sigma_{\gamma \pi^{+}} \sim 150 \mu$ barns (Bhattacharjee \& Sigl 2000). Evaluating $K$ based on the assumed energy density of the cluster, we then find

$$
\begin{aligned}
Q_{\gamma e^{+} e^{-}} & \sim 3 \times 10^{-26} N_{p, 0} T_{\mathrm{keV}}^{1 / 2}\left(\frac{n_{e}}{10^{-3} \mathrm{~cm}^{-3}}\right)^{2} \frac{r_{c}}{200 \mathrm{kpc}} \mathrm{cm}^{-3}, \\
Q_{\gamma \pi^{+}} & \sim 5 \times 10^{-30} N_{p, 0} T_{\mathrm{keV}}^{1 / 2}\left(\frac{n_{e}}{10^{-3} \mathrm{~cm}^{-3}}\right)^{2} \frac{r_{c}}{200 \mathrm{kpc}} \mathrm{cm}^{-3} .
\end{aligned}
$$

Thus, $Q_{\gamma \pi} \ll Q_{\gamma e^{+} e^{-}} \ll Q_{\mathrm{pp}}$, and the two photocollision processes may indeed be neglected in even the most luminous clusters. (We find this conclusion to hold regardless of the detailed photon spectrum assumed, provided that the total energy density is fixed.) Note that despite our conclusion that $Q_{\gamma \pi} \ll Q_{\gamma e^{+} e^{-}}$, photopion production is a more significant energy loss mechanism for high-energy protons than is photopair production. The difference in significance results from cosmic-ray protons with $511 \lesssim \gamma_{p} T_{\mathrm{keV}} \lesssim 8 \times 10^{4}$ being energetic enough to produce pairs (but not pions) and from the greater inelasticity of photopion production as compared to photopair production (Mannheim \& Schlickeiser 1994).

\section{CALCULATION OF THE POSITRON DISTRIBUTION FUNCTION}

\subsection{Positron Injection by an $A G N$}

For positrons that are injected impulsively by a shortlived AGN embedded in the cluster, the source term $Q(\gamma)=0$ in equation (1). If we further neglect annihilation of the cooling positrons, the calculation of the positron distribution function is straightforward (Sarazin 1999). This approximation is valid because for any $\gamma>\gamma_{\mathrm{eq}}$, the annihilation time is much longer than the cooling time. (See $\S 4.1$ for further discussion of this point.)

The calculation proceeds as follows. We first assume that the AGN injects positrons into the cluster with a distribution function $N_{+}\left(\gamma, t_{i}\right)$, where $t_{i}$ is the injection time. Consider a positron with Lorentz factor $\gamma_{i}$ at time $t_{i}$. At a later time $t$, the positron will have cooled to a Lorentz factor $\gamma_{0}$. Number conservation demands that

$$
\int_{\gamma_{0}}^{\infty} N_{+}\left(\gamma^{\prime}, t\right) d \gamma^{\prime}=\int_{\gamma_{i}}^{\infty} N_{+}\left(\gamma^{\prime}, t_{i}\right) d \gamma^{\prime} .
$$

Differentiating, we find (Sarazin 1999)

$$
N_{+}\left(\gamma_{0}, t\right)=\left.N_{+}\left(\gamma_{i}, t_{i}\right) \frac{d \gamma_{i}}{d \gamma_{0}}\right|_{t},
$$

with $\gamma_{i}$ computed using the cooling function $b(\gamma, t)$ given in $\S 2.1$.

At any time $t>t_{i}$, the number of positrons that have not yet cooled $n_{u}(t)$ may be calculated by integrating the distribution function over all Lorentz factors $\gamma>\gamma_{\text {eq. }}$. The number density of thermalized positrons $n_{+}(t)$ then evolves according to

$$
\frac{d n_{+}}{d t}=-\frac{d n_{u}}{d t}-n_{+} A\left(\gamma_{\mathrm{eq}}\right),
$$

where the first term is the rate at which positrons thermalize and the second term is the rate at which they annihilate. Thus,

$$
n_{+}(t)=e^{-A\left(\gamma_{\mathrm{eq}}\right) t} \int_{t_{i}}^{t} d t^{\prime} e^{A\left(\gamma_{\mathrm{eq}}\right) t^{\prime}}\left|\frac{d n_{u}}{d t}\right| .
$$

We calculate the initial distribution function $N_{+}\left(\gamma, t_{i}\right)$ by expressing the total positron energy density (including the rest mass) immediately after injection as

$$
u_{\mathrm{rel}}=m_{e} c^{2} \int \gamma N_{+}\left(\gamma, t_{i}\right) d \gamma=\xi_{\mathrm{AGN}} \frac{L_{K} \tau}{V},
$$

where $L_{K}$ is the total mechanical luminosity of the AGN, $\tau$ is the lifetime of the AGN (in a single duty cycle), $V$ is the volume over which the relativistic particles are mixed, and $\xi_{\mathrm{AGN}}$ is the fraction of the injected energy in the positron component (see below). For typical parameter values, the energy input from a central AGN is significant compared to the thermal energy stored in the cluster core and could, in some cases, balance the energy lost to cooling flow radiation (Böhringer et al. 2002). If we assume that the positrons initially have a power-law distribution in momentum,

$$
\begin{array}{r}
N_{+}\left(\gamma, t_{i}\right)=n_{i} \frac{\gamma}{\left(\gamma^{2}-1\right)^{(s+1) / 2}}, \\
\gamma_{+, \text {min }}<\gamma<\gamma_{+, \text {max }},
\end{array}
$$


the normalization constant $n_{i}$ is

$$
\begin{aligned}
n_{i}= & \frac{3.9 \times 10^{-7}}{C_{s}^{\mathrm{AGN}}} \frac{\xi_{\mathrm{AGN}}}{0.1} \frac{L_{K}}{10^{45} \mathrm{ergs} \mathrm{s}^{-1}} \\
& \times \frac{\tau}{10^{8} \mathrm{yr}}\left(\frac{r_{\mathrm{mix}}}{200 \mathrm{kpc}}\right)^{-3} \mathrm{~cm}^{-3},
\end{aligned}
$$

where $r_{\text {mix }} \sim r_{c}$ is the radius out to which positrons are distributed and $C_{S}^{\mathrm{AGN}}$ depends on the power-law index of the distribution and is determined by equation (32):

$$
\begin{aligned}
& C_{2}^{\mathrm{AGN}}=\ln \left(\frac{\gamma_{+, \max }+\sqrt{\gamma_{+, \max }^{2}-1}}{\gamma_{+, \min }+\sqrt{\gamma_{+, \text {min }}^{2}-1}}\right) \\
& +\frac{\gamma_{+, \min }}{\sqrt{\gamma_{+, \min }^{2}-1}}-\frac{\gamma_{+, \max }}{\sqrt{\gamma_{+, \max }^{2}-1}}, \\
& C_{3}^{\mathrm{AGN}}=\frac{1}{4}\left\{\ln \left[\frac{\left(\gamma_{+, \max }-1\right)\left(\gamma_{+, \text {min }}+1\right)}{\left(\gamma_{+, \max }+1\right)\left(\gamma_{+, \min }-1\right)}\right]\right. \\
& \left.+\frac{\gamma_{+, \min }}{\gamma_{+, \min }^{2}-1}-\frac{\gamma_{+, \max }}{\gamma_{+, \max }^{2}-1}\right\} \text {. }
\end{aligned}
$$

In equation (33), $\gamma_{+, \text {max }} \sim 4 \times 10^{7} M\left(B_{\text {sh, } \mu \mathrm{G}} T_{\mathrm{keV}}\right)^{1 / 2}$ is fixed by equating the acceleration time to the IC cooling time. $B_{\mathrm{sh}}$ thus refers to the magnetic field in the shocks inside the accelerating region of the jet system; for simplicity, we take $B_{\text {sh }}=B$. We note that the magnetic field in the accelerating region may have considerably larger values than the ambient cluster field because of the powerful magnetic fields in AGN jets (e.g., Begelman et al. 1984). However, the dependence on $\gamma_{+, \max }$ is only logarithmic and assuming a larger $B$ value introduces only a small change to our results. We also assume that $M \sim 10$, a value appropriate for the hot-spot velocity near the end of the active phase (see, for example, the model in Furlanetto \& Loeb 2001).

As above, we determine $\gamma_{+, \text {min }}$ assuming that the minimum cosmic-ray momentum is a few times the mean thermal momentum. For positrons, this implies that

$$
\gamma_{+, \min }^{2}-1=0.74 T_{\mathrm{keV}}\left(\frac{c_{1}}{2.3}\right)^{2}\left(\frac{M}{10}\right)^{2} .
$$

Note that $\left(\gamma_{+, \min }-1\right)$ is of order unity for reasonable cluster parameters.

The distribution function in equation (33) should be thought of as referring to the positron component once it eventually escapes the entire region of interaction between the relativistic jets and the ambient medium. It is not necessarily the same distribution the positrons have while inside the jet, where cooling may be efficient (Begelman et al. 1984) but where the positrons are expected to have a bulk Lorentz factor $\gamma_{\text {jet }} \sim 10$. As they travel along the jet and particularly when they pass through the hot spot, surviving positrons will presumably be reaccelerated to a fresh distribution given by equation (33). The normalization factor $\xi_{\mathrm{AGN}}$ thus represents the net fraction of the interaction energy $L_{K} \tau$ transferred to the escaping positrons. Note that we assume that all of the positrons are accelerated by the shocks. If only a fraction are actually accelerated, the total number of positrons (and hence the annihilation signal) would only increase.

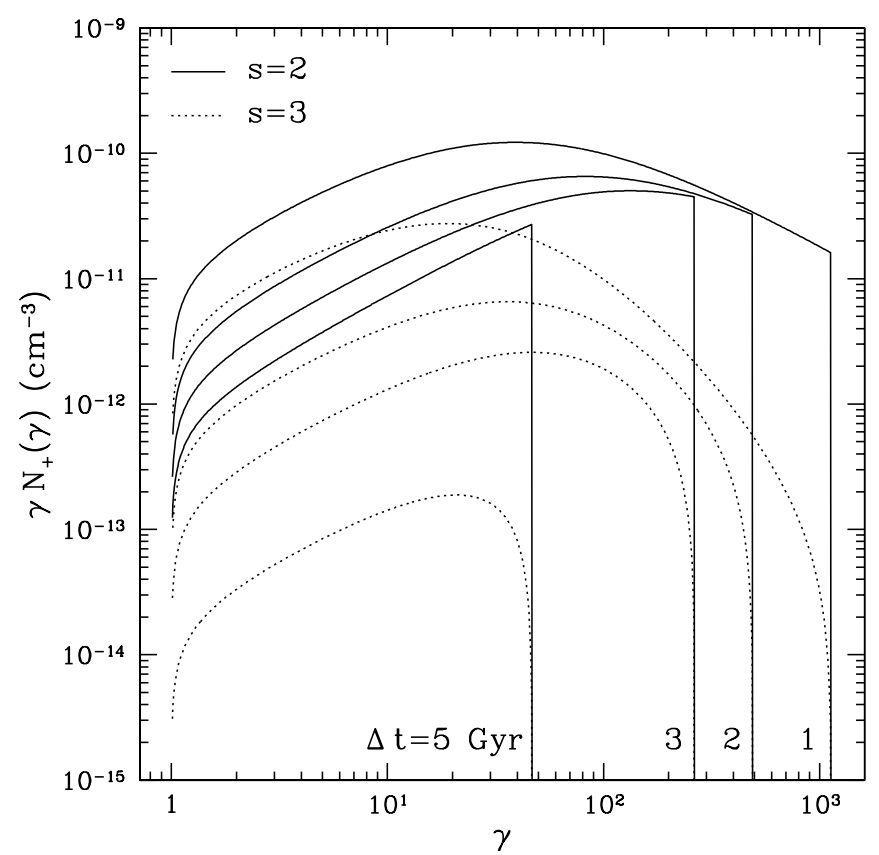

FIG. 2.-Distribution function of nonthermalized positrons $N_{+}(\gamma, t)$ for injection via an AGN at various times. Shown are an $s=2$ injection spectrum (solid curves) and an $s=3$ injection spectrum (dotted curves). In each case, curves correspond to $\Delta t=t_{0}-t_{i}=(1,2,3,5) \times 10^{9} \mathrm{yr}$, from top to bottom. All cases assume standard cluster parameters (see text).

Figure 2 shows $N_{+}(\gamma, t)$ in the AGN scenario at several different time intervals after injection. We present results for an $s=2$ injection spectrum (solid curves) and for an $s=3$ injection spectrum (dotted curves); in each case, curves show $t_{0}-t_{i}=(1,2,3,5) \times 10^{9} \mathrm{yr}$, from top to bottom. Here $t_{0}$ is the (present day) age of the universe in our assumed cosmology. All cases assume what we will refer to as our "standard cluster parameters:" $L_{K}=10^{45} \mathrm{ergs} \mathrm{s}^{-1}$, $\tau=10^{8} \mathrm{yr}, \quad n_{e}=10^{-3} \mathrm{~cm}^{-3}, \quad k_{\mathrm{B}} T_{e}=1 \mathrm{keV}, \quad B=3 \mu \mathrm{G}$, $r_{\text {mix }}=200 \mathrm{kpc}, \quad$ and $\quad \xi_{\mathrm{AGN}}=0.1 . \quad$ Note $\quad$ that $N_{+}(\gamma, t) \propto \xi_{\mathrm{AGN}} L_{K} \tau r_{\text {mix }}^{-3}$, with a more subtle dependence on $n_{e}$ (see below). This scaling holds as long as our approximation of instantaneous positron injection is valid, namely, for $\tau \lesssim 3 \times 10^{8} \mathrm{yr}$. The dependence on temperature enters only through $\gamma_{+ \text {,min. }}$. Because the cooling rate increases with the cluster magnetic field strength, the number of highenergy positrons decreases with $B$. However, our fiducial value of $B=3 \mu \mathrm{G}$ makes $u_{\mathrm{B}} \approx u_{\mathrm{CMB}}$, so lowering the magnetic field strength below this value cannot decrease the cooling rate by more than a factor of 2 (see eq. [4]). This choice for $B$ is motivated by Faraday rotation measurements of clusters (Kim et al. 1990; Clarke et al. 2001). If instead $B \sim 0.1 \mu \mathrm{G}$, as inferred by assuming that hard $\mathrm{X}$ ray excesses in clusters are due to IC emission (Rephaeli et al. 1999; Fusco-Femiano et al. 1999), our final results change only slightly (see $\S 4.2$ for more on this point).

The main feature in the distribution function is the sharp cutoff at Lorentz factor $\gamma_{\max }(t)$. At any given time after injection, this corresponds to

$$
\gamma_{\max }(t)=\lim _{\gamma_{i} \rightarrow \infty} \gamma\left(\gamma_{i}, t, t_{i}\right),
$$

where $\gamma\left(\gamma_{i}, t\right)$ is the Lorentz factor at time $t$ of a positron injected with $\gamma_{i}$ at time $t_{i}$. The limit is well defined because 


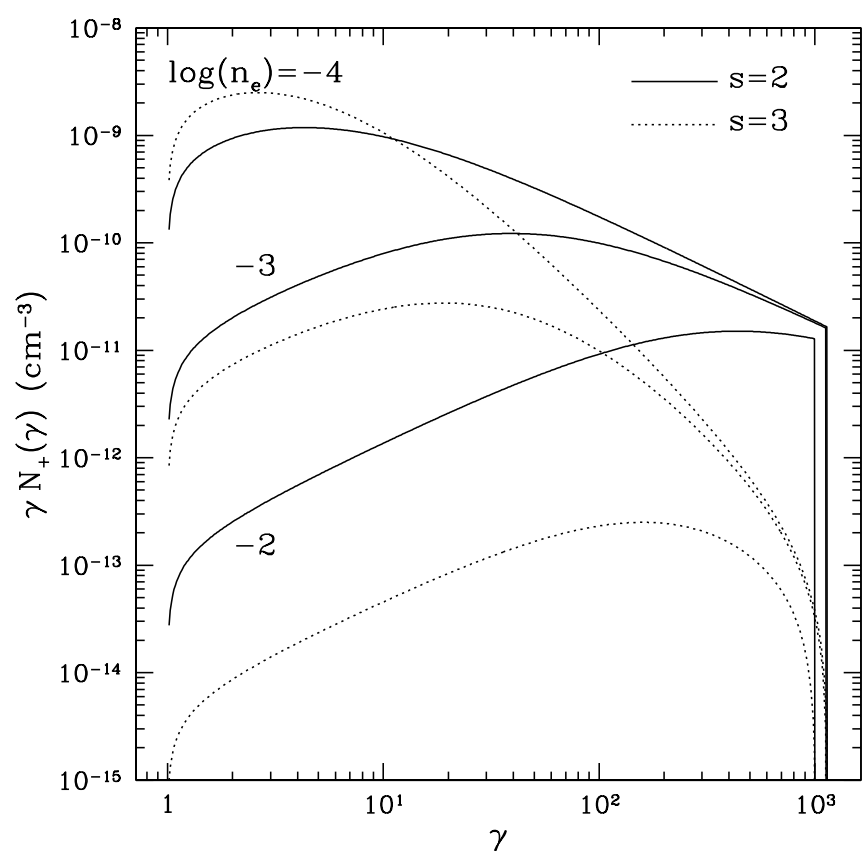

FIG. 3.-Distribution function of nonthermalized positrons $N_{+}(\gamma, t)$ for injection via an AGN at various ambient densities. Shown are an $s=2$ injection spectrum (solid curves) and an $s=3$ injection spectrum (dotted curves). In each case, curves correspond to $n_{e}=10^{-4}, 10^{-3}$, and $10^{-2} \mathrm{~cm}^{-3}$, from bottom to top. All cases assume $t_{0}-t_{i}=10^{9} \mathrm{yr}$ and standard cluster parameters (with the exception of $n_{e}$; see text).

$b(\gamma) \propto \gamma^{2}$ for large Lorentz factors; however, we do not solve for it in closed form here. Sarazin (1999) gives expressions for $\gamma_{\max }(t)$ in some simple limits.

Figure 3 shows the effects of varying the cluster core density $n_{e}$ on the distribution function of the uncooled positrons. Again, the solid (dotted) curves show results for an $s=2$ (3) injection spectrum. All assume $t_{0}-t_{i}=10^{9} \mathrm{yr}$ and, aside from the density, standard cluster parameters. From top to bottom, the curves in each case assume $n_{e}=10^{-4}, 10^{-3}$, and $10^{-2} \mathrm{~cm}^{-3}$. Perhaps surprisingly, $N_{+}(\gamma)$ decreases as $n_{e}$ increases. This nonintuitive behavior is a result of the cooling rates. Above the cooling "bottleneck" at $\gamma \sim 200\left(n_{e} / 10^{-3} \mathrm{~cm}^{-3}\right)^{1 / 2}$, the cooling time is independent of density (see eq. [5]); below this Lorentz factor, the cooling time is inversely proportional to density (see eq. [7]). In other words, once positrons enter the Coulomb cooling regime, they thermalize at a rate proportional to $n_{e}$. Thus, near the bottleneck $N_{+}(\gamma)$ is independent of density, while below it $N_{+}(\gamma)$ actually decreases with increasing density because positrons enter the thermalized component faster. (This density dependence does not hold for the thermalized positron density $n_{+}$.)

The evolution of $n_{+}$as a function of cosmic time is shown in the bottom panels of Figures 4 and 5. Figure 4 shows results for an $s=2$ positron injection spectrum; the solid curves assume $n_{e}=10^{-3} \mathrm{~cm}^{-3}$ with $t_{0}-t_{i}=1,2,3$, and $5 \times 10^{9} \mathrm{yr}$, while the dotted curve assumes $n_{e}=10^{-4} \mathrm{~cm}^{-3}$ with $t_{0}-t_{i}=5 \times 10^{9} \mathrm{yr}$, and the dashed curve assumes $n_{e}=10^{-2} \mathrm{~cm}^{-3}$ with $t_{0}-t_{i}=5 \times 10^{9}$ yr. Figure 5 shows a similar set of results for an $s=3$ injection spectrum. In all cases, we assume standard cluster parameters (except for the electron density). We find that steepening the power-law slope $s$ increases the number of thermalized positrons because the total number of positrons produced by the

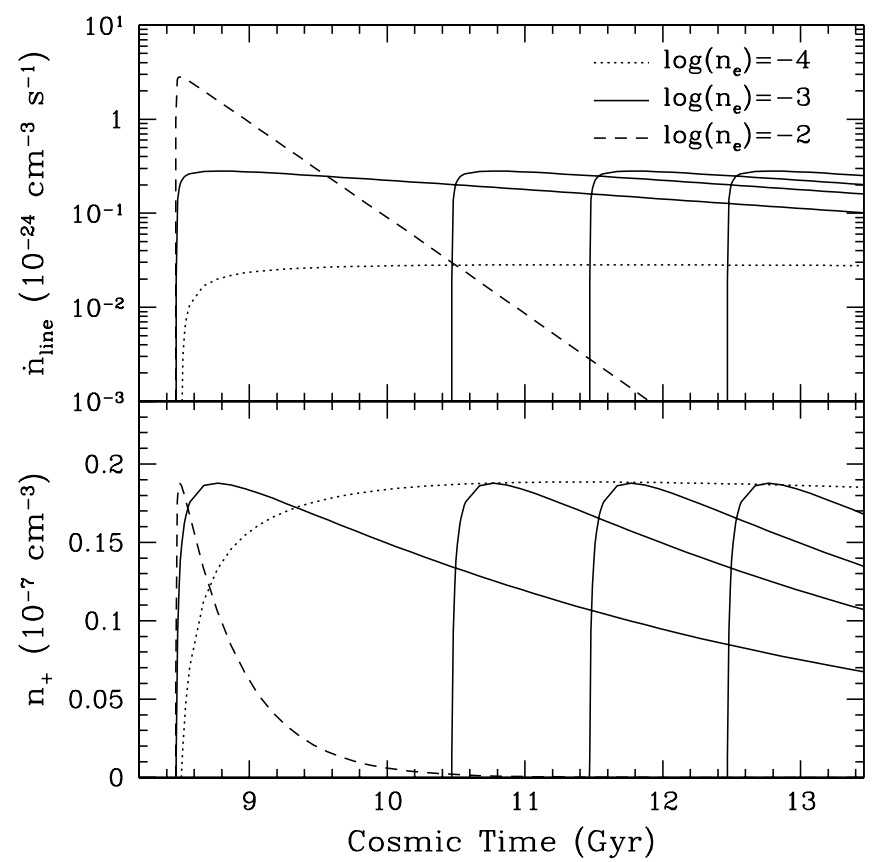

Fig. 4.-Evolution of the thermalized positron density $n_{+}$(bottom panel) and of the emissivity in the annihilation line $\dot{n}_{\text {line }}$ (top panel) as a function of cosmic time for an $s=2$ positron injection spectrum in the AGN case. Shown are results for $n_{e}=10^{-3} \mathrm{~cm}^{-3}$ with $t_{0}-t_{i}=1,2,3$, and $5 \times 10^{9} \mathrm{yr}$ from left to right (solid curves), $n_{e}=10^{-4} \mathrm{~cm}^{-3}$ with $t_{0}-t_{i}=5 \times 10^{9} \mathrm{yr} \quad$ (dotted curve), and $n_{e}=10^{-2} \mathrm{~cm}^{-3}$ with $t_{0}-t_{i}=5 \times 10^{9} \mathrm{yr}$ (dashed curve). All curves assume standard cluster parameters (with the exception of $n_{e}$; see text).

AGN increases as $s$ increases. Note that $n_{+}$changes by $\$ 2 \%$ if we decrease the magnetic field strength to $B \sim 0.1 \mu \mathrm{G}$.

At very early times after injection, the thermalization rate exceeds the annihilation rate and $n_{+}$rises rapidly, particu-

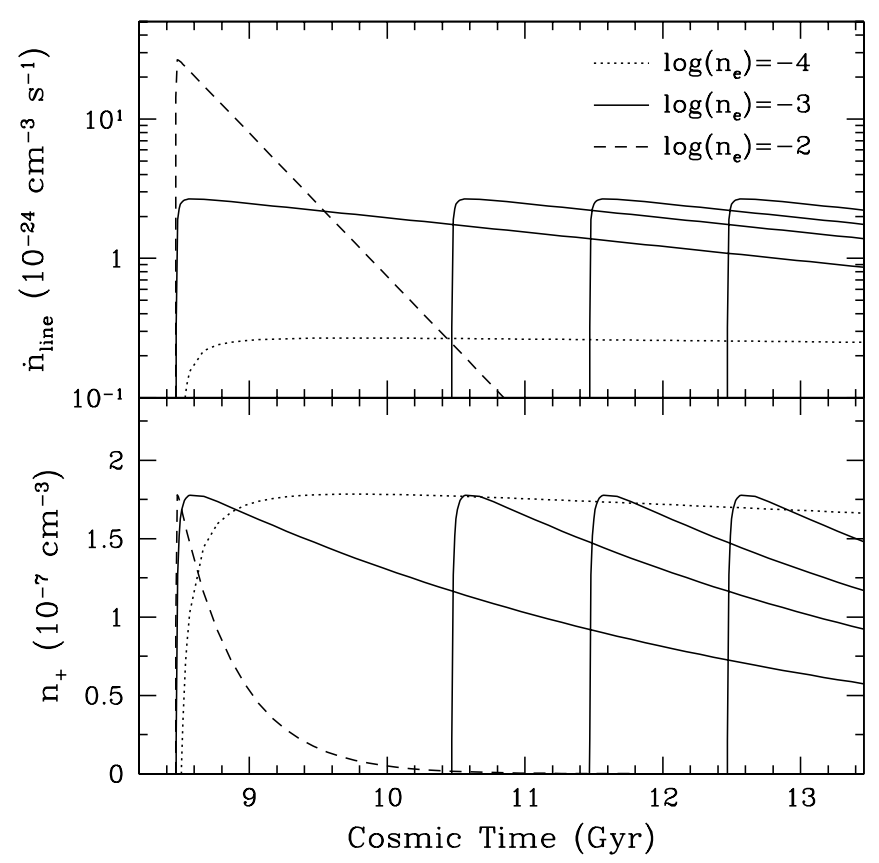

FIG. 5.-Same as Fig. 4, but with an $s=3$ positron injection spectrum 
larly for a steep injection spectrum in which the number density of particles is weighted heavily toward small $\gamma$. However, once the low- $\gamma$ positrons cool, the thermalization rate falls rapidly with time (because fewer positrons begin with large Lorentz factors) and annihilation begins to dominate. Therefore, at late times $n_{+} \propto e^{-A\left(\gamma_{\mathrm{eq}}\right)\left(t-t_{i}\right)}$.

The dependence on density is more complex. The total number density of injected positrons (and hence the maximum value of $n_{+}$) is independent of $n_{e}$, as is the peak value of $n_{+}$. However, because $b_{\text {Coul }} \propto n_{e}, n_{+}$peaks later for smaller ambient densities. Furthermore, the annihilation timescale is $\tau_{\mathrm{ann}} \sim A\left(\gamma_{\mathrm{eq}}\right)^{-1} \sim 4 \times 10^{9}\left(n_{e} / 10^{-3} \mathrm{~cm}^{-3}\right)^{-1} \mathrm{yr}$; for $n_{e}=10^{-4} \mathrm{~cm}^{-3}, \tau_{\mathrm{ann}} \gtrsim H_{0}^{-1}$ and $n_{+}$does not change substantially over the lifetime of the cluster.

Note that the distribution functions derived in this section depend weakly on the cosmological redshift through the corresponding dependence of the IC cooling rate.

\subsection{Steady Injection by Cosmic Rays}

In the case of steady injection, with $Q_{\mathrm{pp}}(\gamma)$ computed as in $\S 2.3 .1$, we can calculate the positron distribution function reached at steady state (i.e., when the total production rate of positrons equals the annihilation rate). In this case, all quantities in equation (1) are independent of time, and the distribution function of uncooled positrons is

$$
N_{+}(\gamma)=\frac{1}{\mu(\gamma)} \int_{\gamma}^{\infty} d \gamma^{\prime} \mu\left(\gamma^{\prime}\right) \frac{Q\left(\gamma^{\prime}\right)}{b\left(\gamma^{\prime}\right)},
$$

where

$$
\mu(\gamma)=\exp \int_{\gamma_{1}}^{\gamma} \frac{d \gamma^{\prime}}{b\left(\gamma^{\prime}\right)}\left[\frac{d b}{d \gamma^{\prime}}-A\left(\gamma^{\prime}\right)\right],
$$

and $\gamma_{1}$ is an arbitrary constant. Note that if we neglect annihilation, this reduces to the equilibrium distribution found by Sarazin (1999). The number density of thermalized positrons may then be found by requiring the total number density of positrons to be independent of time:

$$
n_{+}=\frac{1}{A\left(\gamma_{\mathrm{eq}}\right)} \int_{\gamma_{\mathrm{eq}}}^{\infty} d \gamma\left[Q_{\mathrm{pp}}(\gamma)-N_{+}(\gamma) A(\gamma)\right]
$$

Our scheme requires that enough time has elapsed since the cosmic-ray proton component first formed for the annihilations to reach equilibrium with production. Because the annihilation cross section decreases rapidly with $\gamma_{+}$, this essentially requires that the thermalized component has grown enough that $A\left(\gamma_{\mathrm{eq}}\right) n_{+} \approx Q_{\mathrm{pp}}$. This requires a time $\tau_{\text {eq }} \sim \tau_{\text {ann }}$. We therefore expect equilibrium to be a satisfactory assumption for $n_{e} \gtrsim 3 \times 10^{-4} \mathrm{~cm}^{-3}$, a condition well satisfied by rich clusters.

Figure 6 shows the equilibrium positron distribution $N_{+}(\gamma)$ with $Q_{\mathrm{pp}}(\gamma)$ from $\S 2.3 .1$. We show results for an $s=2$ cosmic-ray proton spectrum (solid curves) and for an $s=3$ proton spectrum (dotted curves). In each case, curves correspond to $k_{\mathrm{B}} T_{e}=1,5$, and $10 \mathrm{keV}$, from bottom to top. All assume $n_{e}=10^{-3} \mathrm{~cm}^{-3}, B=3 \mu \mathrm{G}$, and $\xi_{\text {cr }}=0.1$. The distribution function is strictly proportional to $\xi_{\mathrm{cr}}$. Although $T_{e}$ does affect $\gamma_{p \text {,min }}$ and hence the fraction of cosmic-ray protons able to produce pions, we find that $N_{+}(\gamma)$ is still very nearly proportional to $T_{e}$.

Figure 7 illustrates how $N_{+}(\gamma)$ depends on the density of the ambient medium. As before, the solid (dotted) curves

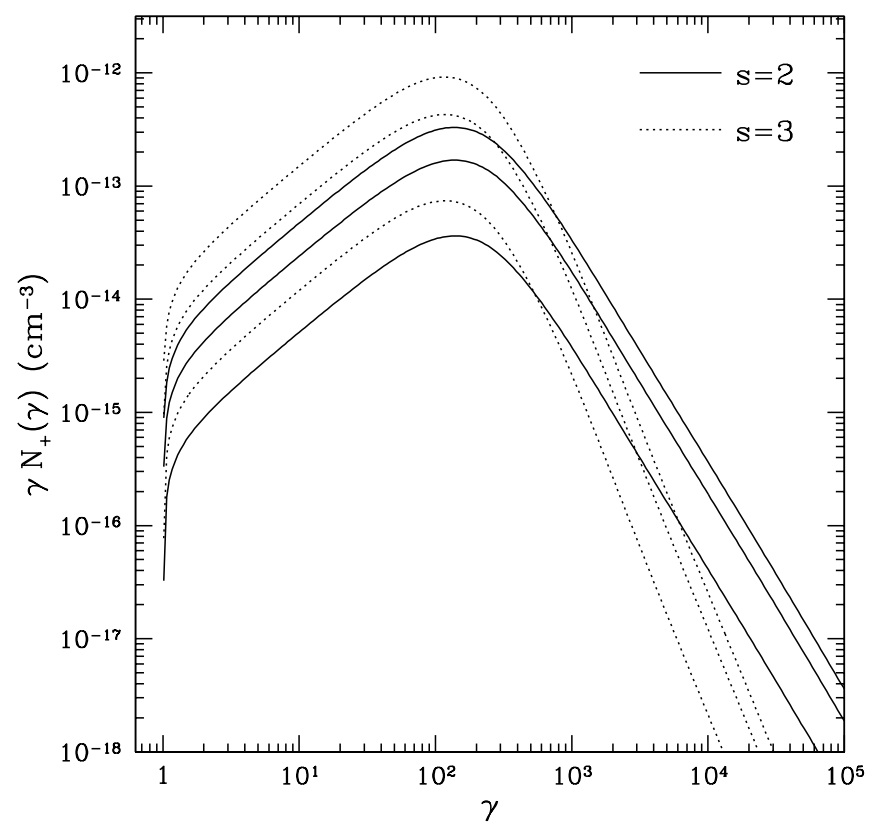

FIG. 6.-Equilibrium distribution function of nonthermalized positrons $N_{+}(\gamma)$ assuming steady $Q_{\mathrm{pp}}(\gamma)$, for various cluster temperatures. Shown are an $s=2$ proton spectrum (solid curves) and an $s=3$ proton spectrum (dotted curves). In each case, curves correspond to $k_{\mathrm{B}} T_{e}=1,5$, and 10 $\mathrm{keV}$, from bottom to top. All curves assume $n_{e}=10^{-3} \mathrm{~cm}^{-3}, B=3 \mu \mathrm{G}$, and $\xi_{\mathrm{cr}}=0.1$.

show results for an $s=2$ (3) proton spectrum. The cluster is assumed to have $\xi_{\mathrm{cr}}=0.1, B=3 \mu \mathrm{G}$, and $k_{\mathrm{B}} T_{e}=10 \mathrm{keV}$, with $n_{e}=10^{-4}, 10^{-3}$, and $10^{-2} \mathrm{~cm}^{-3}$, from bottom to top. We see that the peak in the distribution function moves to larger Lorentz factors as density increases and Coulomb

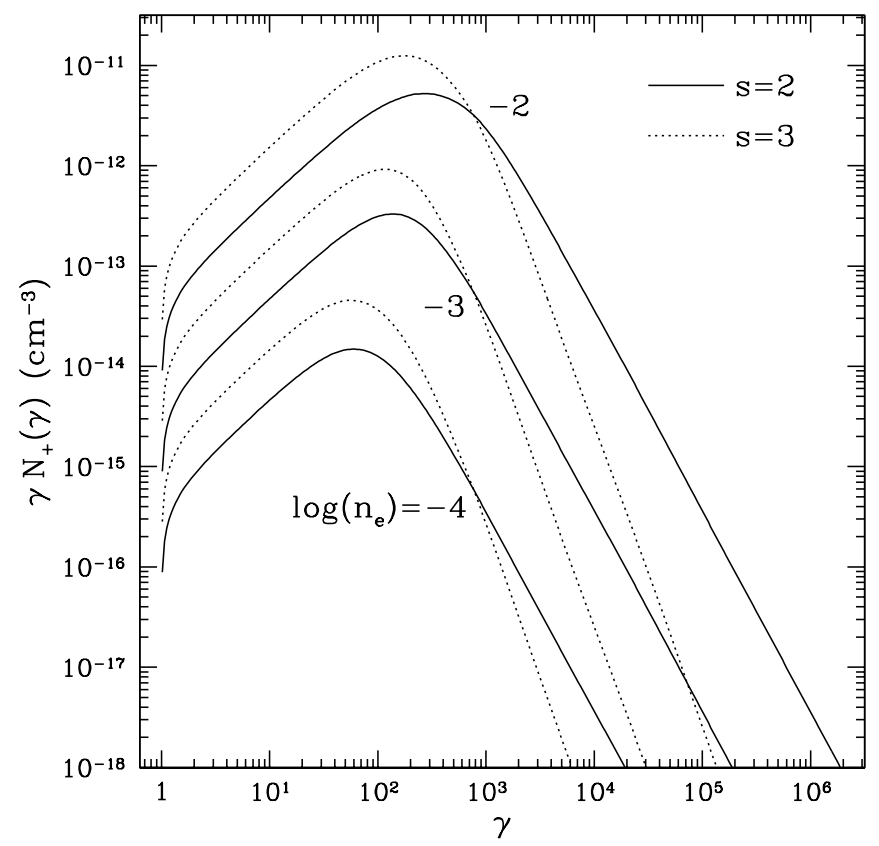

FIG. 7.-Equilibrium distribution function of nonthermalized positrons $N_{+}(\gamma)$ assuming steady $Q_{\mathrm{pp}}(\gamma)$, for various cluster densities. Shown are an $s=2$ proton spectrum (solid curves) and an $s=3$ proton spectrum (dotted curves). In each case, curves correspond to $n_{e}=10^{-4}, 10^{-3}$, and $10^{-2} \mathrm{~cm}^{-3}$, from bottom to top. All curves assume $k_{\mathrm{B}} T_{e}=10 \mathrm{keV}$, $B=3 \mu \mathrm{G}$, and $\xi_{\mathrm{cr}}=0.1$. 
cooling becomes more efficient. Note, however, that the cluster with $n_{e}=10^{-4} \mathrm{~cm}^{-3}$ may not yet have reached steady state.

In all cases, $N_{+}(\gamma) \propto \gamma^{-(s+1)}$ at high energies where synchrotron/IC cooling dominates and annihilation can be neglected (Sarazin 1999). The distribution function peaks where the efficiency of both synchrotron/IC and Coulomb cooling is low $(\gamma \sim 100)$ and then falls again at small Lorentz factors as Coulomb cooling takes over. The location of the peak therefore depends on the assumed cluster magnetic field. Again, however, because our fiducial value of $B=3 \mu \mathrm{G}$ makes IC cooling slightly more efficient than synchrotron cooling, decreasing the magnetic field below this value has only a small effect on $N_{+}(\gamma)(\operatorname{see} \S 4.2)$.

The equilibrium density of thermalized positrons $n_{+}$is not shown in Figures 6 and 7 because it scales simply with the cluster parameters:

$$
n_{+} \sim 8 \times 10^{-14} X_{s}^{\mathrm{cr}} T_{\mathrm{keV}} \frac{n_{e}}{10^{-3} \mathrm{~cm}^{-3}} \frac{\xi_{\mathrm{cr}}}{0.1} \mathrm{~cm}^{-3}
$$

where $X_{s}^{\mathrm{cr}}=1(2.5)$ for an $s=2(s=3)$ proton spectrum.

\section{ANNIHILATION SPECTRUM}

In the following subsections, we calculate the emissivity of annihilation photons from the ICM using equations (10) and (13) for both the AGN ( $\S 4.1)$ and the steady injection (§ 4.2) scenarios. In each case, we use the distribution function $N_{+}(\gamma)$ found in the appropriate subsection of $\S 3$.

\subsection{Positron Injection by an $A G N$}

For injection by an AGN, we ignore annihilation of nonthermalized positrons when calculating $N_{+}(\gamma, t)$, although we do include annihilation of the thermalized component. Here, we calculate the instantaneous emissivity expected for the resulting distribution.

Figure 8 shows the annihilation emissivity $\left(d n_{\gamma} / d \epsilon_{\gamma} d t\right)$ for the same cases as in Figure 2. The continuum, in which the emissivity is approximately $\propto \epsilon_{\gamma}^{-1}$, is generated by the uncooled positrons, while the line is generated by the thermalized positrons. The sharp cutoffs in the continuum occur because of our cosmic-ray approximation in which $\gamma_{-}=1$. In this approximation, only photons with $\left[\gamma_{+, \max }(1-\right.$ $\left.\left.\beta_{+, \max }\right)+1\right] / 2 \leq \epsilon_{\gamma} / m_{e} c^{2} \leq\left[\gamma_{+, \max }\left(1+\beta_{+, \max }\right)+1\right] / 2$ can be generated (Svensson 1982); in reality, the nonzero highenergy tails of the ambient electron distribution (and collisions with relativistic cosmic-ray electrons) will cause the cutoffs to be somewhat smoother. The time evolution of line emissivity is discussed in detail below.

Figure 8 justifies our approach of neglecting annihilation in calculating the distribution function. Consider, for example, the case in which $t_{0}-t_{i}=10^{9} \mathrm{yr}$ for an $s=2$ injection spectrum. The total annihilation emissivity in the continuum is $\sim 5 \times 10^{-28} \mathrm{~cm}^{-3} \mathrm{~s}^{-1}$, yielding a total number of annihilations of nonthermalized positrons since injection of $\sim 8 \times 10^{-12} \mathrm{~cm}^{-3}$. We calculate the total density of nonthermalized positrons to be $\sim 2 \times 10^{-10} \mathrm{~cm}^{-3}$, indicating that only $\sim 5 \%$ of the positrons annihilate before cooling.

The line emissivity at a given time is proportional to $\xi_{\mathrm{AGN}} L_{K} \tau r_{\text {mix }}^{-3}$, and so the total luminosity of the cluster is independent of $r_{\text {mix }}$. The dependence on ambient density is somewhat more complicated. This is displayed in Figure 9, which shows the annihilation emissivity for clusters with

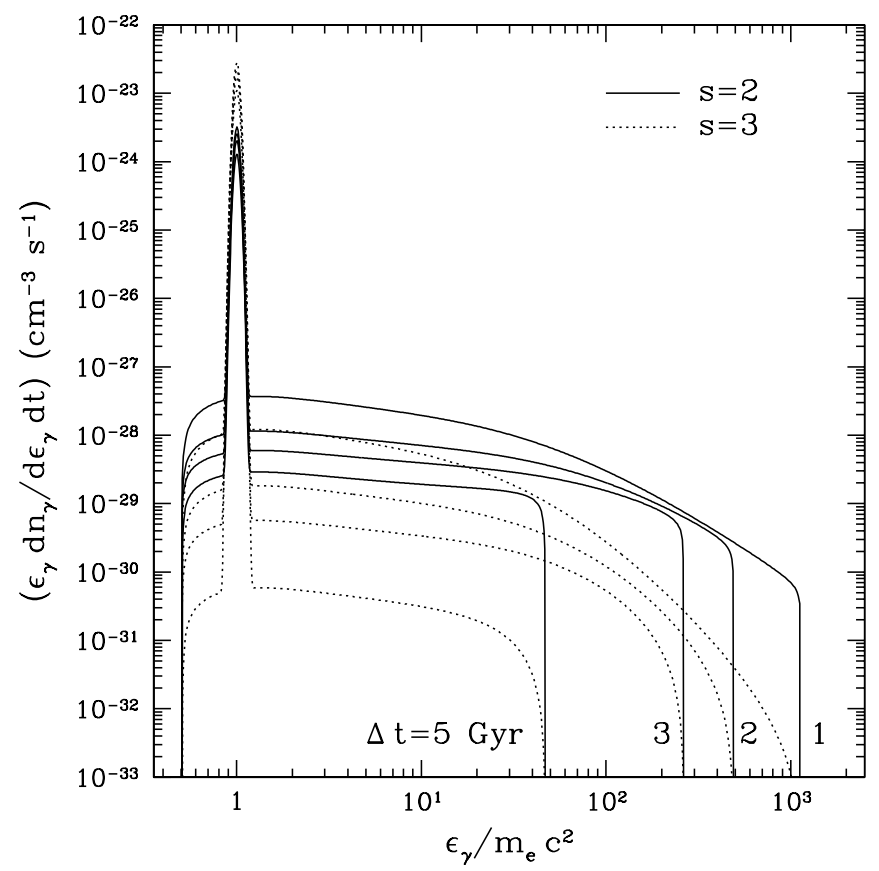

FIG. 8.-Annihilation emissivity $\left(d n_{\gamma} / d \epsilon_{\gamma} d t\right)$ for injection via an AGN at various times. Shown are an $s=2$ injection spectrum (solid curves) and an $s=3$ injection spectrum (dotted curves). In each case, curves correspond to $\Delta t=t_{0}-t_{i}=(1,2,3,5) \times 10^{9} \mathrm{yr}$, from top to bottom in continuum level. All curves assume standard cluster parameters (see text).

densities $n_{e}=10^{-4}$ and $n_{e}=10^{-2} \mathrm{~cm}^{-3}$ for $s=2$ and $s=3$ positron input spectra (solid and dotted curves, respectively). Standard cluster parameters (with the exception of density) are assumed, with $t_{0}-t_{i}=10^{9} \mathrm{yr}$. The continuum emissiv-

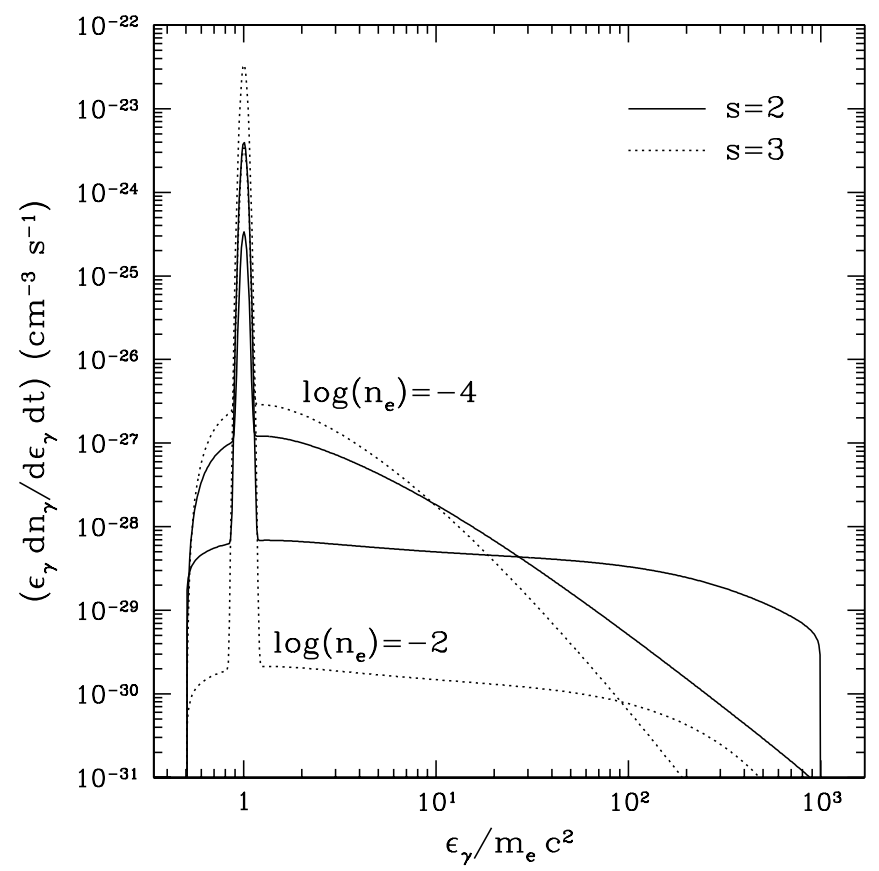

FIG. 9.-Annihilation emissivity $\left(d n_{\gamma} / d \epsilon_{\gamma} d t\right)$ for injection via an AGN at various ambient densities. Shown are an $s=2$ injection spectrum (solid curves) and an $s=3$ injection spectrum (dotted curves). In each case, we show results for $n_{e}=10^{-4}$ and $10^{-2} \mathrm{~cm}^{-3}$. All curves assume standard cluster parameters (with the exception of $n_{e}$; see text) and $t_{0}-t_{i}=10^{9} \mathrm{yr}$. 


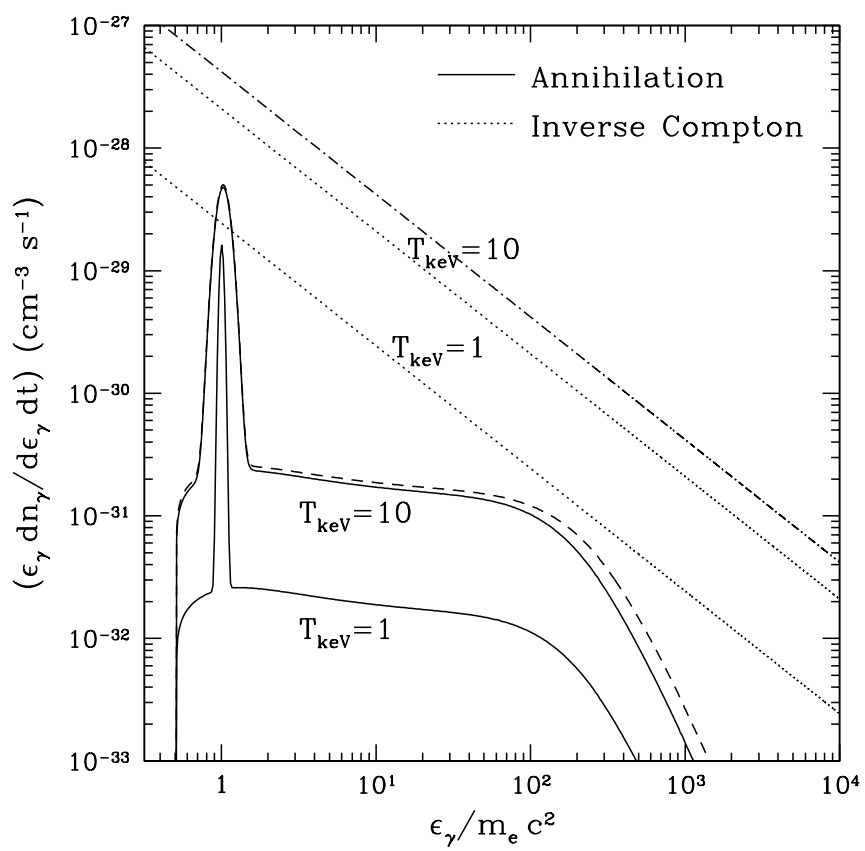

FIG. 10.- Spectral emissivity $\left(d n_{\gamma} / d \epsilon_{\gamma} d t\right)$ assuming steady $Q_{\mathrm{pp}}(\gamma)$ for an $s=2$ proton spectrum. Solid curves show the annihilation emissivity for clusters at $k_{\mathrm{B}} T_{e}=1 \mathrm{keV}$ (bottom) and $k_{\mathrm{B}} T_{e}=10 \mathrm{keV}$ (top). Dotted curves show the inverse Compton emissivity determined from $N_{+}(\gamma)$ for the same temperatures. These curves assume $n_{e}=10^{-3} \mathrm{~cm}^{-3}, B=3 \mu \mathrm{G}$, and $\xi_{\mathrm{cr}}=0.1$. We also show annihilation and IC emissivities for $B=0.1 \mu \mathrm{G}$ and $k_{\mathrm{B}} T_{e}=10 \mathrm{keV}$ (dashed and dot-dashed curves, respectively).

ity depends on the shape of $N_{+}(\gamma, t)$, which has a nonnegligible dependence on $n_{e}$. Note that more continuum photons are produced in lower density media. This is because, as shown in Figure 3, a larger fraction of the positrons have thermalized by this epoch in high-density clusters, and the thermalized component contributes only to the line emission. The dependence of the peak line luminosity on density is very different from that of the continuum (see below). The cluster temperature affects both the normalization of the continuum (although only relatively weakly) and the width of the annihilation line (the FWHM is $\sim 32 T_{\mathrm{keV}}^{1 / 2} \mathrm{keV}$; see eq. [13]). This temperature-dependent line broadening is displayed graphically in Figure 10 below. As described above, the cluster magnetic field has only a small effect on $n_{+}$and hence on the annihilation line for reasonable values of the field strength (see the discussion in $\S 4.2$; the magnitude of the effect is similar in this case).

Observationally, the critical observable is the emissivity of the annihilation line; this quantity varies strongly as a function of the source age. We show the evolution of the line emissivity $\dot{n}_{\text {line }}$ with time in the top panels of Figures 4 and 5 for the same cases shown in the bottom panels. The emissivity is proportional to the number of thermalized positrons, so at late times $\dot{n}_{\text {line }} \propto n_{e} e^{-A\left(\gamma_{\text {eq }}\right)\left(t-t_{i}\right)}$. Thus, the peak signal decreases as density decreases but it also fades with a timescale $\tau_{\text {ann }} \propto n_{e}^{-1}$.

The emissivities calculated in this section depend weakly on redshift through the IC cooling rate.

\subsection{Steady Injection by Cosmic Rays}

In the steady injection scenario, we have calculated $N_{+}(\gamma)$ self-consistently, including annihilation. The resultant photon emissivities are shown in Figures 10 and 11 for

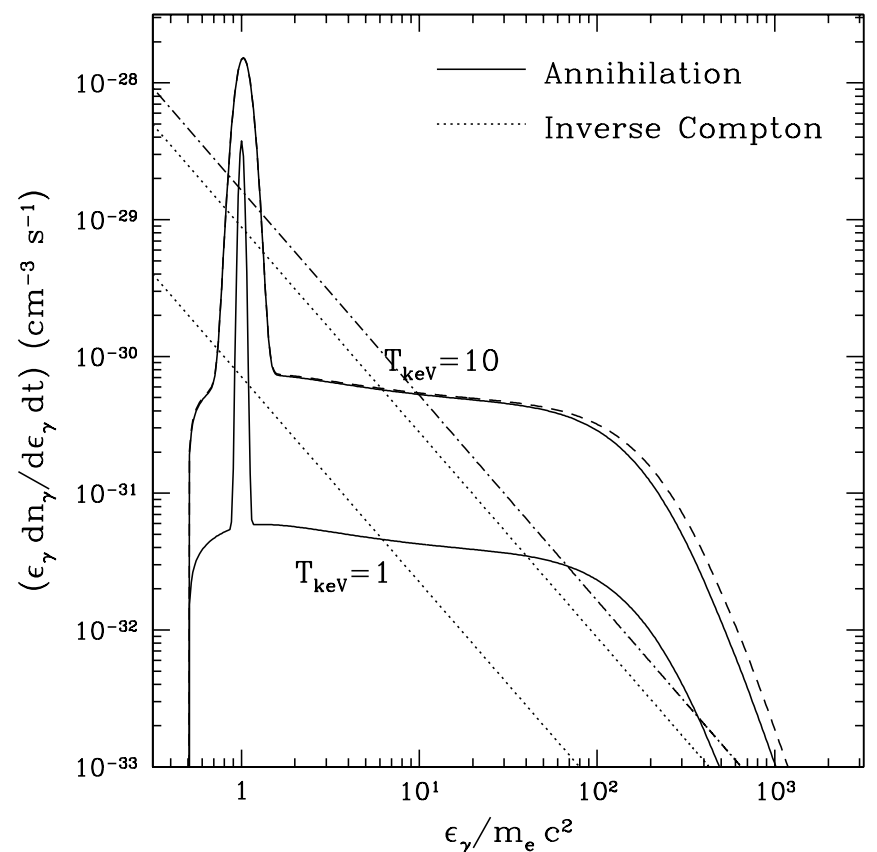

Fig. 11.-Same as Fig. 10, but with an $s=3$ proton injection spectrum.

$s=2$ and $s=3$ proton spectra, respectively. In each case, the solid curves show the annihilation emissivity, with $k_{\mathrm{B}} T_{e}=1 \mathrm{keV}$ (bottom) and $k_{\mathrm{B}} T_{e}=10 \mathrm{keV}($ top $)$.

In these figures, we also show the inverse Compton emissivity determined from the equilibrium positron spectrum $N_{+}(\gamma)$. In the energy range of interest $\left(\gamma \approx 10^{4}\right), N_{+}(\gamma)$ has assumed its asymptotic power-law form, and the inverse Compton spectrum can be estimated easily (Rybicki \& Lightman 1979):

$$
\begin{aligned}
\left.\epsilon_{\gamma} \frac{d n_{\gamma}}{d \epsilon_{\gamma} d t}\right|_{\mathrm{IC}} \approx & 5 \times 10^{-20} I_{s} T_{\mathrm{keV}} \frac{\xi_{\mathrm{cr}}}{0.1}\left(\frac{n_{e}}{10^{-3} \mathrm{~cm}^{-3}}\right)^{2} \\
& \times\left(\frac{k_{\mathrm{B}} T_{\mathrm{CMB}}}{\epsilon_{\gamma}}\right)^{s / 2} \mathrm{~cm}^{-3} \mathrm{~s}^{-1},
\end{aligned}
$$

where $I_{s}=1\left(10^{3}\right)$ for an $s=2(s=3)$ proton spectrum. This is shown in Figures 10 and 11 by the dotted lines. Clearly, the IC emission from the positrons themselves is a substantial contaminant and in fact hides the annihilation radiation completely for relatively shallow proton spectra. Thus, only if the protons are accelerated by very weak shocks (so that $s \sim 3$ ) is the annihilation radiation from the secondary positrons observable even in principle.

In the steady injection case, $\left(d n_{\gamma} / d \epsilon_{\gamma} d t\right) \propto N_{p, 0} n_{e} \propto \xi_{\mathrm{cr}} n_{e}^{2}$. The ambient density also determines the energy at which the continuum begins to decline rapidly; the break is located at $\epsilon_{\gamma} \approx \gamma_{\mathrm{pk}} m_{e} c^{2}$, where $\gamma_{\mathrm{pk}}$ is the location of the peak of $N_{+}(\gamma)$ and is determined by the efficiency of Coulomb cooling. The temperature affects the normalization of the continuum and line $\left(\dot{n}_{\text {line }} \propto T_{e}\right)$ as well as the determination of the width of the line component, as described in the previous subsection.

Figures 10 and 11 also show explicitly the effects of lowering the cluster magnetic field to $B=0.1 \mu \mathrm{G}$ (dashed curves) along with the corresponding IC emission (dot-dashed curves), assuming $k_{\mathrm{B}} T_{e}=10 \mathrm{keV}$. This is the magnetic field strength inferred from hard X-ray excesses in clusters, pro- 
vided that they are due to IC emission (e.g., Rephaeli et al. 1999; Fusco-Femiano et al. 1999). We find that the weaker magnetic field enhances the high-energy positron population over that in the fiducial model by about a factor of 2 . However, because the number density of high-energy positrons is so small, this has virtually no effect on the annihilation line. It does increase the strength of the continuum tail and the IC background by a factor of $\sim 2$. We see that our predictions for the annihilation line are largely independent of the cluster magnetic field, and even if reconciling the hard $\mathrm{X}$-ray observations with the relatively high magnetic field strengths inferred from Faraday rotation measurements requires highly inhomogeneous magnetic fields, our results will be accurate to within a few percent. We note in passing that even for an unrealistically high average $B \sim 30 \mu \mathrm{G}$, the annihilation emissivity changes by $\lesssim 10 \%$.

\section{DISCUSSION}

We have calculated the signals expected from positron annihilation in galaxy clusters for positrons injected as primaries by embedded radio jets and those produced as secondaries in collisions between cosmic rays and thermal protons in the cluster. The former case is of interest in constraining the matter content of relativistic jets, while the latter is of interest in measuring the cosmic-ray content of the ICM. The positron annihilation line is particularly interesting for these purposes because typical cluster temperatures $k_{\mathrm{B}} T_{e} \sim 1-10 \mathrm{keV}$ are just in the range in which positrons annihilate efficiently without forming positronium. We calculate the annihilation rates assuming that the cooling positrons mix efficiently with the ambient medium on cosmological timescales.

For positron injection by a single AGN, the peak emissivity in the annihilation line is

$$
\begin{aligned}
\dot{n}_{\text {line, } \text { single }} \approx & 2 \times 10^{-25} X_{s}^{\mathrm{AGN}} \frac{n_{e}}{10^{-3} \mathrm{~cm}^{-3}} \frac{\xi_{\mathrm{AGN}}}{0.1}\left(\frac{r_{\text {mix }}}{200 \mathrm{kpc}}\right)^{-3} \\
& \times \frac{L_{K}}{10^{45} \mathrm{ergs} \mathrm{s}^{-1}} \frac{\tau}{10^{8} \mathrm{yr}} \mathrm{cm}^{-3} \mathrm{~s}^{-1}
\end{aligned}
$$

(single AGN event), with $X_{s}^{\mathrm{AGN}}=1$ (10) for an $s=2$ (3) positron injection spectrum. The dependence on $s$ appears primarily because, with the normalization procedure described in $\S 3.1$, the total number of $e^{+}$produced increases as $s$ increases. We note that rich clusters can have core densities $n_{e} \sim 10^{-2} \mathrm{~cm}^{-3}$ and that $\xi_{\mathrm{AGN}}$ may reach a value of several tens of percent for jets with a low bulk Lorentz factor. The time lag between injection and peak annihilation depends principally on the electron density $n_{e}$ in the cluster core; the characteristic annihilation time of the positron population is $\tau_{\text {ann }} \sim 4 \times 10^{9}\left(n_{e} / 10^{-3} \mathrm{~cm}^{-3}\right)^{-1} \mathrm{yr}$, and the emissivity maintains the peak level for roughly this time period (see Figs. 4 and 5). Therefore, although dense clusters produce the strongest signals, they fade relatively quickly.

The longevity of the positron population in typical clusters indicates that the signal can be enhanced if we consider either multiple injection epochs or multiple AGNs in a single cluster. A model of the former type has been advocated recently by Böhringer et al. (2002) as a way of balancing the cooling flow radiation in some clusters. These authors suggest quasi-periodic mechanical energy injection from the central galaxy throughout the lifetime of the cluster, with injection epochs lasting $\sim 10^{8} \mathrm{yr}$ occurring every $\Delta t_{\text {inj }} \sim 10^{9}$ yr. In the limit in which $\tau_{\text {ann }} \gg \Delta t_{\text {inj }}$, the annihilation emissivity will reach a quasi-steady state with $\dot{n}_{\text {line }} \sim \eta^{-1} \dot{n}_{\text {line,single }}$, where $\eta=\Delta t_{\text {inj }} / \tau_{\text {ann }}$ is the period between outbursts in units of the annihilation time of the cluster. Here $L_{K}$ and $\tau$ are to be interpreted as the mechanical luminosity and lifetime of a single outburst.

An even more interesting possibility is positron injection by galaxies throughout the cluster. While the vast majority of these galaxies are not active at the present day, recent studies have found evidence for relic supermassive black holes in nearly all bulge-dominated galaxies (Magorrian et al. 1998; Gebhardt et al. 2000). This, together with modeling of the quasar luminosity function, suggests that nearly all galaxies once hosted a quasar (Haiman \& Loeb 1998; Haehnelt \& Kauffmann 2000). Current observations give the relation $M_{\mathrm{BH}}=7.8 \times 10^{7}\left(L_{\mathrm{B}, \text { bulge }} / 10^{10} L_{\mathrm{B}, \odot}\right)^{1.08} M_{\odot}$ (Kormendy \& Gebhardt 2001). Because the observed black hole mass-bulge luminosity relation is nearly linear, for the purposes of an estimate it suffices to assume a linear relation and scale our results with the total cluster core luminosity $L_{\mathrm{B}, \mathrm{cl}}$. A typical rich cluster core has a total $B$-band luminosity of $L_{\mathrm{B}, \mathrm{cl}} \sim 10^{12} L_{\mathrm{B}, \odot}$ (Peebles 1993). (This luminosity corresponds to $\sim 100 L_{\mathrm{B}}^{*}$ galaxies in the cluster, where $L_{\mathrm{B}}^{*}$ is the characteristic luminosity of galaxies in the Schechter function; Yasuda et al. 2001.) We further assume a mass-toenergy conversion efficiency $\varepsilon_{\mathrm{BH}}$ during the black hole formation process and that a fraction $f_{K}$ of this energy is released in outflows. We expect $\varepsilon_{\mathrm{BH}} \sim 0.1$, and observations indicate that $f_{K} \sim 0.1$ (Hooper et al. 1995; Furlanetto \& Loeb 2001, and references therein). Therefore, if we make the extreme assumption that all injection events occur simultaneously, the peak line emissivity would be

$$
\begin{aligned}
\dot{n}_{\text {line }} \approx & 9 \times 10^{-23} X_{s}^{\mathrm{AGN}} \frac{n_{e}}{10^{-3} \mathrm{~cm}^{-3}} \frac{\xi_{\mathrm{AGN}}}{0.1}\left(\frac{r_{\text {mix }}}{200 \mathrm{kpc}}\right)^{-3} \\
& \times\left(\frac{f_{K}}{0.1} \frac{\varepsilon_{\mathrm{BH}}}{0.1} \frac{L_{\mathrm{B}, \mathrm{cl}}}{10^{12} L_{\mathrm{B} \odot}}\right) \mathrm{cm}^{-3} \mathrm{~s}^{-1}
\end{aligned}
$$

(multiple simultaneous AGNs), with $X_{S}^{\mathrm{AGN}}$ defined as above. Of course, we must keep in mind that the quasar era peaked at $z \sim 2$ (Pei 1995; in our cosmology, $t_{0}-t_{i} \sim 10^{10} \mathrm{yr}$ ) so a substantial fraction of the population may have annihilated before the present day. In the opposite limit, in which the source evolution time is much larger than the annihilation time but in which the annihilation time is in turn much larger than the time between injection events (or $H_{0}^{-1} \gg \tau_{\text {ann }} \gg \Delta t_{\text {inj }}$ ), a quasi-steady state will be reached as described in the previous paragraph, with a steady annihilation emissivity of approximately $\eta^{-1}$ times that of a typical $\mathrm{AGN}$ in the cluster.

For positron production by cosmic rays, the steady state emissivity in the annihilation line is

$$
\dot{n}_{\text {line }} \approx 10^{-30} X_{s}^{\mathrm{cr}} T_{\mathrm{keV}}\left(\frac{n_{e}}{10^{-3} \mathrm{~cm}^{-3}}\right)^{2} \frac{\xi_{\mathrm{cr}}}{0.1} \mathrm{~cm}^{-3} \mathrm{~s}^{-1},
$$

(cosmic-ray secondaries), with $X_{s}^{\mathrm{cr}}=1$ (2.5) for an $s=2$ $(s=3)$ proton spectrum. For an $s=2$ input spectrum, the energy lost to positron annihilation over the age of the universe is $\sim 10^{-6} U_{\mathrm{cr}}\left(n_{e} / 10^{-3} \mathrm{~cm}^{-3}\right)$, where $U_{\mathrm{cr}}$ is the cosmicray energy of the cluster. The emission mechanism is inefficient because $\lesssim 10^{-3}$ of the cosmic-ray energy goes into the 
positrons and because the characteristic initial energy of the positrons is $\gg m_{e} c^{2}$ (see Fig. 1), so most of the initial positron energy is lost to cooling radiation before annihilation occurs. We therefore see that direct positron injection by even a weak $\operatorname{AGN}\left(L_{K} \gtrsim 10^{41} \mathrm{ergs} \mathrm{s}^{-1}\right)$ would overwhelm the signal from secondary positrons produced by cosmicray protons.

The observability of the line is of course the critical question. The flux at earth from a cluster with a positron mixing radius $r_{\text {mix }}$ is

$$
\begin{aligned}
F_{c}= & 8 \times 10^{-7}\left(\frac{r_{\text {mix }}}{200 \mathrm{kpc}}\right)^{3}\left(\frac{D}{100 \mathrm{Mpc}}\right)^{-2} \\
& \times \frac{\dot{n}_{\text {line }}}{10^{-24} \mathrm{~cm}^{3} \mathrm{~s}^{-1}} \text { photons } \mathrm{cm}^{-2} \mathrm{~s}^{-1},
\end{aligned}
$$

where $D$ is the luminosity distance to the cluster. In the AGN injection case, the emissivity is $\propto r_{\text {mix }}^{-3}$, so the flux is independent of the mixing scale (rather, it depends on the total number of positrons injected by the AGN). For steady production by cosmic-ray protons, the emissivity is independent of the volume of the cluster core and the flux scales in proportion to the mixing volume.

Before discussing the prospects for detection of this signal with upcoming instruments, we must consider possible contaminating backgrounds. First, as described in the previous subsection, the relativistic positrons (and electrons) with $\gamma \gtrsim 10^{4}$ in the cluster generate IC radiation at $511 \mathrm{keV}$. However, cooling depletes this population rapidly; after a time interval $t-t_{i} \sim 1.5 \times 10^{8} \mathrm{yr}$, the maximum Lorentz factor $\gamma_{\max }<10^{4}$ and the electrons and positrons can no longer produce IC radiation at $511 \mathrm{keV}$. However, in the case of steady injection from cosmic-ray protons, the IC background originates from the equilibrium distribution itself. Figures 10 and 11 show that in this case, the IC background can overwhelm the annihilation line unless the protons have a sufficiently steep $(s \sim 3)$ injection spectrum.

Second, we must also consider the diffuse extragalactic background, for which the flux at $\epsilon_{\gamma} \approx 511 \mathrm{keV}$ is $F_{\text {bkgd }} \sim 2 \times 10^{-5}$ photons $\mathrm{cm}^{-2} \mathrm{~s}^{-1} \mathrm{keV}^{-1} \mathrm{sr}^{-1}$ (Watanabe et al. 1997). For a cluster of a fixed size, the ratio of the line flux from the cluster to the background flux is independent of cluster distance so long as $z \ll 1$ :

$$
\frac{F_{c}}{F_{\text {bkgd }}} \sim 10^{2} T_{\mathrm{keV}}^{-1 / 2} \frac{r_{\text {mix }}}{200 \mathrm{kpc}} \frac{\dot{n}_{\text {line }}}{10^{-24} \mathrm{~cm}^{-3} \mathrm{~s}^{-1}} .
$$

Thus, the expected signal from AGN-injected positrons is well above the diffuse background. However, the signal from secondary positrons generated by cosmic rays will be hidden by the background.

While the prospects for observing the annihilation of secondary positrons in the foreseeable future are small (even if the annihilation line can be observed over the IC background), positrons injected by AGN may soon be detectable with space-borne instruments. The International GammaRay Astrophysical Laboratory (INTEGRAL), ${ }^{2}$ expected to be launched in 2002 October, will have spectral capabilities in the energy range of interest. The SPI instrument is expected to have a $3 \sigma$ line sensitivity $\sim 5.1 \times 10^{-6}$ photons html. $\mathrm{cm}^{-2} \mathrm{~s}^{-1}$ given an integration time of $10^{6} \mathrm{~s}$, but its poor angular resolution (2.5) may lead to background contamination. The IBIS instrument, with $12^{\prime}$ resolution, is better suited to cluster detection, but it has a $3 \sigma$ line sensitivity of only $\sim 10^{-4}$ photons $\mathrm{cm}^{-2} \mathrm{~s}^{-1}$ (again for an integration time of $10^{6} \mathrm{~s}$ ). An even more powerful search could be conducted with the Energetic X-Ray Imaging Survey Telescope $(E X I S T),{ }^{3}$ a proposed all-sky hard X-ray survey mission. It has an expected $5 \sigma$ line sensitivity of $\sim 5 \times 10^{-6}$ photons $\mathrm{cm}^{-2} \mathrm{~s}^{-1}$ in the relevant energy range (assuming an integration time of $10^{7} \mathrm{~s}$, the mean exposure time planned for any point on the sky in the mission), and it has an excellent angular resolution of $5^{\prime}$. All of these instruments should be able to resolve the annihilation line. IBIS will have a spectral resolution of $\sim 10 \mathrm{keV}$, SPI will have a resolution $\sim 1.5 \mathrm{keV}$, and EXIST will have a resolution $\sim 3 \mathrm{keV}$. The expected line width is $\sim 32 T_{\mathrm{keV}}^{1 / 2} \mathrm{keV}$, well above these limits for typical clusters. If the positrons are injected by AGNs, the sensitivity limits are close to the signal we predict for nearby $(D \lesssim 100 \mathrm{Mpc}$ ) clusters with powerful AGNs, multiple injection epochs/galaxies, or steep injection spectra. Deep exposures with INTEGRAL or statistical analyses taking advantage of the full-sky coverage of EXIST may reveal weaker sources as well.

A particularly interesting source is the nearby Virgo cluster at a distance $\sim 20 \mathrm{Mpc}$. In the AGN scenario, the annihilation signal in Virgo is comparable to the detection threshold of all three instruments listed above. The expected peak flux is

$$
\begin{aligned}
F_{\text {Virgo }} \approx & 1.3 \times 10^{-6} X_{s}^{\mathrm{AGN}} \eta^{-1} \frac{n_{e}}{3 \times 10^{-3} \mathrm{~cm}^{-3}} \frac{\xi_{\mathrm{AGN}}}{0.1} \\
& \times \frac{L_{K}}{10^{44} \mathrm{ergs} \mathrm{s}^{-1}} \frac{\tau}{10^{8} \mathrm{yr}} \text { photons cm } \mathrm{cm}^{-2} \mathrm{~s}^{-1}
\end{aligned}
$$

where we have used fiducial values for the luminosity of M87 estimated by Böhringer et al. (2002) and for $n_{e}$ by Nulsen \& Böhringer (1995). Here, $\eta^{-1}$ represents the contribution from past AGN phases of cluster galaxies (see the discussion accompanying eq. [45]); in the best case, it could represent an enhancement of more than an order of magnitude. If $r_{\text {mix }}$ is large, the high-resolution instruments may even be able to map spatial variations in the positron component (and indicate whether the positrons are injected solely by the central galaxy or by a larger number of galaxies during the quasar era).

Continuum $\gamma$-ray emission from M87, the dominant galaxy in Virgo, may contaminate the annihilation line signal. To date there have been no observations of Virgo in the relevant energy range. To estimate the contamination from M87, we assume that the observed power-law spectrum in the 2-10 keV range extends to $511 \mathrm{keV}$; using the recent observations of Böhringer et al. (2001), we expect M87 to produce a flux in the spectral regime of the annihilation line of $\sim 4 \times 10^{-8} T_{\mathrm{keV}}^{1 / 2}$ photons $\mathrm{cm}^{-2} \mathrm{~s}^{-1}$ (the temperature of the core of Virgo varies with radius between $k_{\mathrm{B}} T_{e}=1$ and 3 $\mathrm{keV}$; see Böhringer et al. 2001). Thus, even if the continuum emission from M87 cannot be removed through the use of a high angular resolution instrument, the annihilation line should still be visible. Of course, because M87 is still active, any positrons produced in the current outburst phase have

\footnotetext{
${ }^{3}$ See http:/ /exist.gsfc.nasa.gov.
} 
most likely not had sufficient time to cool. We would therefore expect annihilation line emission only if there is a relic population of positrons from either earlier outbursts of M87 or the other galaxies in Virgo.

Another interesting source is Centaurus A, the nearest $(D \approx 3.5 \mathrm{Mpc})$ bright radio galaxy to the Milky Way. The radio structure is one of the largest in both apparent and absolute size, covering an area $8^{\circ} \times 4^{\circ}$ on the sky (or $480 \times 240 \mathrm{kpc}$ ) and is therefore a very attractive candidate for observations (Israel 1998). The expected flux in the positron annihilation line is

$$
\begin{aligned}
F_{\mathrm{Cen} \mathrm{A}} \approx & 10^{-5} X_{s}^{\mathrm{AGN}} \eta^{-1} \frac{n_{e}}{10^{-2} \mathrm{~cm}^{-3}} \frac{\xi_{\mathrm{AGN}}}{0.1} \\
& \times \frac{L_{K}}{10^{43} \mathrm{ergs} \mathrm{s}^{-1}} \frac{\tau}{1.4 \times 10^{8} \mathrm{yr}} \text { photons } \mathrm{cm}^{-2} \mathrm{~s}^{-1}
\end{aligned}
$$

where we have scaled $L_{K}$ to the approximate bolometric luminosity of the Centaurus A nuclear source (Chiaberge, Capetti, \& Celotti 2001) and $\tau$ to the minimum source age estimated by Saxton, Sutherland, \& Bicknell (2001). Recent data from the Chandra X-Ray Observatory indicates that the gas density around Centaurus $\mathrm{A}$ is $n_{e} \approx 10^{-2}(r /$ $5 \mathrm{kpc})^{-1.33} \mathrm{~cm}^{-3}$ at $0.5 \mathrm{kpc} \ll r \lesssim 10 \mathrm{kpc}$ (R. P. Kraft et al. 2002 , in preparation). The signal is potentially stronger than that of M87; however, several caveats are in order. First, the gas near the central source has a temperature $k_{\mathrm{B}} T_{e} \sim 0.275 \pm 0.03 \mathrm{keV}$ (R. P. Kraft et al. 2002, in preparation) within the regime in which positronium formation begins to play a significant role. More importantly, the scale of the radio emission suggests that a large fraction of the positrons escape the interstellar medium of Centaurus A and mix with the intragroup medium at $r \gtrsim 100 \mathrm{kpc}$. The inferred virial temperature of the group is only $\sim 0.07 \mathrm{keV}$ (based on the observed velocity dispersion of group galaxies; van den Bergh 2000). At this temperature, the positronium formation rate is approximately equal to the free annihilation rate (Crannell et al. 1976). The gas density will also be much lower at large radii (if the observed power-law decline at $r \sim 10 \mathrm{kpc}$ continues, then $n_{e} \sim 2 \times 10^{-4} \mathrm{~cm}^{-3}$ at $r \sim 100 \mathrm{kpc})$. Therefore, it is unclear whether annihilation in this region can produce a strong line. In addition, because the source is still active, the positrons may not yet have had time to cool or mix sufficiently with the surrounding gas. Finally, because the Centaurus A group contains only $\sim 30$ galaxies (van den Bergh 2000), additional positron enrichment from other AGNs is likely to be small.

In summary, we have shown that although there are a variety of mechanisms for producing positrons in galaxy clusters, only direct injection by AGNs is likely to produce an observable signal. Therefore, we argue that a positive detection of positron annihilation lines from clusters suspected of harboring dormant AGNs would be a robust indication that radio jets contain an $e^{+} e^{-}$pair plasma.

We thank J. Grindlay and C. Canizares for helpful comments on the manuscript and R. Kraft for providing unpublished data on Centaurus A. This work was supported in part by NASA grants NAG 5-7039 and 5-7768 and by NSF grants AST 99-00877 and AST 00-71019 (for A. L.). S. R. F. acknowledges the support of an NSF graduate fellowship.

\section{REFERENCES}

Badhwar, G. D., Golden, R. L., \& Stephens, S. A. 1977, Phys. Rev. D, 15, 820

Begelman, M. C., Blandford, R. D., \& Rees, M. J. 1984, Rev. Mod. Phys., 56, 255

Bell, A. R. 1978, MNRAS, 182, 147

Berestetskii, V. B., Lifshitz, E. M., \& Pitaevskii, L. P. 1982, Quantum Electrodynamics (New York: Pergamon)

Berezinsky, V. S., Blasi, P., \& Ptuskin, V. S. 1997, ApJ, 487, 529

Bhattacharjee, P., \& Sigl, G. 2000, Phys. Rep., 327, 109

Blandford, R. D., \& Eichler, D. 1987, Phys. Rep., 154, 1

Blandford, R. D., \& Ostriker, J. P. 1978, ApJ, 221, L29

Blasi, P. 1999, ApJ, 525, 603

Blasi, P., \& Colafrancesco, S. 1999, Astropart. Phys., 12, 169

Boettcher, M., \& Schlickeiser, R. 1996, A\&A, 306, 86

Böhringer, H., et al. 2001, A\&A, 365, L181

Böhringer, H., Matsushita, K., Churazov, E., Ikebe, Y., \& Chen, Y. 2002, A\&A, 382, 804

Butt, Y. M., Torres, D. F., Combi, J. A., Dame, T., \& Romero, G. E. 2001, ApJ, 562, L167

Chiaberge, M., Capetti, A., \& Celotti, A. 2001, MNRAS, 324, L33

Chodorowski, M. J., Zdziarski, A. A., \& Sikora, M. 1992, ApJ, 400, 181

Clarke, T. E., Kronberg, P. P., \& Böhringer, H. 2001, ApJ, 547, L111

Colafrancesco, S., \& Blasi, P. 1998, Astropart. Phys., 9, 227

Crannell, C. J., Joyce, G., Ramaty, R., \& Werntz, C. 1976, ApJ, 210, 582

Dermer, C. D. 1986, ApJ, 307, 47

Dolag, K., \& Enßlin, T. A. 2000, A\&A, 362, 151

Ellison, D. C., Berezhko, E. G., \& Baring, M. G. 2000, ApJ, 540, 292

Fields, B. D., Olive, K. A., Cassé, M., \& Vangioni-Flam, E. 2001, A\&A, 370,623

Furlanetto, S. R., \& Loeb, A. 2001, ApJ, 556, 619

Fusco-Femiano, R., et al. 1999, ApJ, 513, L21

Gebhardt, K., et al. 2000, ApJ, 543, L5

Gieseler, U. D. J., Jones, T. W., \& Kang, H. 2000, A\&A, 364, 911

Ginzburg, V. L., \& Syrovatskii, S. I. 1964, The Origin of Cosmic Rays (New York: Macmillan)

Haehnelt, M. G., \& Kauffmann, G. 2000, MNRAS, 318, L35

Haiman, Z., \& Loeb, A. 1998, ApJ, 503, 505

Hooper, E. J., Impey, C. D., Foltz, C. B., \& Hewett, P. C. 1995, ApJ, 445, 62

Israel, F. P. 1998, A\&A Rev., 8, 237

Kang, H., \& Jones, T. W. 1995, ApJ, 447, 944

Kempner, J. C., \& Sarazin, C. L. 2001, ApJ, 548, 639

Kim, K.-T., Kronberg, P. P., Dewdney, P. E., \& Landecker, T. L. 1990, ApJ, 355, 29

Kinzer, R. L., et al. 2001, ApJ, 559, 282

Kormendy, J., \& Gebhardt, K. 2001, in AIP Conf. Ser. 586, 20th Texas Symp. on Relativistic Astrophysics, ed. H. Martel \& J. C. Wheeler (Melville: AIP), 363

Koyama, K., et al. 1995, Nature, 378, 255 -1997, PASJ, 49, L7

Maciolek-Niedzwiecki, A., Zdziarski, A. A., \& Coppi, P. S. 1995, MNRAS, 276, 273

Magorrian, J., et al. 1998, AJ, 115, 2285

Mannheim, K., \& Schlickeiser, R. 1994, A\&A, 286, 983

Miniati, F. 2001, Comput. Phys. Commun., 141, 17

Miniati, F., Jones, T. W., Kang, H., \& Ryu, D. 2001a, ApJ, 562, 233

Miniati, F., Ryu, D., Kang, H., \& Jones, T. W. 2001b, ApJ, 559, 59

Mirabel, I. F. 2001, Ap\&SS, 276, 319

Moskalenko, I. V., \& Strong, A. W. 1998, ApJ, 493, 694

Muraishi, H., et al. 2000, A\&A, 354, L57

Nulsen, P. E. J., \& Böhringer, H. 1995, MNRAS, 274, 1093

Peebles, P. J. E. 1993, Principles of Physical Cosmology (Princeton: Princeton Univ. Press)

Pei, Y. C. 1995, ApJ, 438, 623

Rephaeli, Y. 1979, ApJ, 227, 364

Rephaeli, Y., Gruber, D., \& Blanco, P. 1999, ApJ, 511, L21

Rybicki, G. B., \& Lightman, A. P. 1979, Radiative Processes in Astrophysics (New York: Wiley)

Sarazin, C. L. 1999, ApJ, 520, 529

Sarazin, C. L., \& Kempner, J. C. 2000, ApJ, 533, 73

Saxton, C. J., Sutherland, R. S., \& Bicknell, G. V. 2001, ApJ, 563, 103

Stecker, F. W. 1970, Ap\&SS, 6, 377

Svensson, R. 1982, ApJ, 258, 321

Svensson, R., Larsson, S., \& Poutanen, J. 1996, A\&AS, 120, C587

Tanimori, T., et al. 1998, ApJ, 497, L25

Trubnikov, B. A. 1965, in Reviews of Plasma Physics, Vol. 5 (New York: Consultants Bureau), 105

van den Bergh, S. 2000, AJ, 119, 609

Wang, J.-M., Durouchoux, P., \& Li, T.-P. 2001, Ap\&SS, 276, 301

Watanabe, K., et al. 1997, in AIP Conf. Proc. 410, The Fourth Compton Symposium, ed. C. D. Dermer, M. S. Strickman, \& J. D. Kurfess (Woodbury: AIP Press), 1223

Yasuda, N., et al. 2001, AJ, 122, 1104 